\title{
Real Number Computability and Domain Theory*
}

\author{
Pietro Di Gianantonio \\ dipartimento di Matematica e Informatica, Università di Udine \\ via delle Scienze 206 I-33100 Udine Italy \\ E-mail: pietro@dimi.uniud.it
}

\begin{abstract}
We present the different constructive definitions of real number that can be found in the literature. Using domain theory we analyse the notion of computability that is substantiated by these definitions and we give a definition of computability for real numbers and for functions acting on them. This definition of computability turns out to be equivalent to other definitions given in the literature using different methods.

Domain theory is a useful tool to study higher order computability on real numbers. An interesting connection between Scott-topology and the standard topologies on the real line and on the space of continuous functions on reals is stated. An important result in this paper is the proof that every computable functional on real numbers is continuous w.r.t. the compact open topology on the function space.
\end{abstract}

\section{Introduction}

Turing in 1937 was the first to introduce the notion of computable real number [Tur37]. Since then a great number of different approaches have been used to investigate, from a constructive standpoint, main concepts arising in analysis such as real number, limit, derivative and measure. These enterprises have been referred to with various names, e.g., recursive analysis, constructive analysis and computable analysis.

Although the theory of computable analysis can be considered a well-developed subject, there have been so far very few attempts of implementing computable analysis on digital computers, see Boehm and Cartwright, Grue, Vuillemin, [BCRO86], [Boe87], [Gru88], [Vui88]. Such implementations should lead to the realization of "exact real number computation".

${ }^{*}$ Work partially supported by MURST $40 \%$ grant, by EEC/HCM Network "Lambda Calcul Typé" and by an SERC Senior Fellowship. An earlier version of this paper appeared in the Proceedings of the 18th International Symposium on Mathematical Foundation of Computer Science 1993. 
In ordinary practice the computation on real numbers is performed by approximating real numbers by a subset of the rational numbers and by approximating the arithmetic on real numbers by a limited precision arithmetic on rationals. In exact real computation instead the result of a computation can be obtained with arbitrary precision, thus getting rid of the unfortunate phenomenon of the "round-off error".

In this work we do not face directly the problem of defining a feasible effective implementation of exact real number computation. We go instead towards the direction of closing the gap existing between the theory of computable analysis and actual computation. In order to study computability over real numbers we use several tools peculiar to the theory of programming languages. In particular we use domain theory to give a notion of computability on real numbers. This approach turns out to be very fruitful for several reasons. We discuss the adequacy of Scott-domains as domains for representing real numbers. In the literature on real number computation different kinds of partial orders have been employed. We relate the Scott-topology on such domains to the Euclidean topology on $\mathbb{R}$. Using the theory of effective Scott-domains we obtain simpler proofs of some of the classical results of constructive analysis. Domain theory turns out to be useful also in the study of higher order functions. In particular one of the most important results contained in this work concerns the characterisation of the topological properties of the computable higher order functions on reals.

The outline of the paper is as follows. In section 2 we give a survey of the different forms of real number representations used in computable analysis. In section 3 we present a domain that can be used to study real numbers. This domain follows the approach of constructing approximation spaces for the real numbers. This construction has a domain theoretic interest. In fact it is the first example of the use of Scott-domains in an area where normally continuous cpo's (i.e., retracts of algebraic cpo's) are used. In section 4 we investigate the connection between the Scott-topology and the Euclidean topology on the real line. Moreover we present several important and original results that describe the topological properties of computable real functions. The significance of these results lies in the possibility of characterising the topological properties of the computable higher order functions.

\section{Real Number Representations}

Since the seminal work of Turing, a great number of different approaches have been used to study constructive analysis. An important difference between these approaches lies in the way real numbers are represented. Different representations already occur in classical analysis: Cauchy sequences of rational numbers, Cauchy sequences of decimal rationals, Dedekind cuts in the field of rationals, infinite decimal expansions, and so on. Classically all these representations are equivalent and we can study Analysis without worrying about which representation for real numbers we are currently using. Also in computable Analysis 
many of these representations turn out to be equivalent. But there are also some exceptions: for instance Dedekind cuts and Cauchy sequences turn out not to be equivalent.

Between the various constructive representations of real numbers in use there is one that can be considered the most general and taken as a reference. In this representation a real number is defined as the limit of a computable sequence of rational intervals. A formalization of the notion of a computable sequence of rational intervals is given by the following definition.

Notation Let $\langle\langle\rangle$ be any effective coding of pairs of natural numbers and let and $\{\mid\}$ be any effective coding of finite subsets of natural numbers.

Definition 1 Enumeration functions for integers, rationals and rational intervals are defined respectively as:

$\epsilon_{Z}\left(\left\langle\left\langle n_{1}, n_{2}\right\rangle\right\rangle\right)=n_{1}-n_{2}$

$\epsilon_{Q}\left(\left\langle\left\langle n_{1}, n_{2}\right\rangle\right\rangle\right)=\epsilon_{Z}\left(n_{1}\right) /\left(n_{2}+1\right)$

$\epsilon_{I}\left(\left\langle\left\langle n_{1}, n_{2}\right\rangle\right\rangle\right)=\left[\epsilon_{Q}\left(n_{1}\right), \epsilon_{Q}\left(n_{1}\right)+\left|\epsilon_{Q}\left(n_{2}\right)\right|\right]$

A sequence of rational intervals (naturals, integers, rationals) $\left\langle s_{0}, \ldots s_{i} \ldots\right\rangle$ is computable if there is a recursive function $f: \mathbb{N} \rightarrow \mathbb{N}$ s.t.

$\forall i \in \mathbb{N} . s_{i}=\epsilon_{I}(f(i)) \quad\left(=f(i),=\epsilon_{Z}(f(i)),=\epsilon_{Q}(f(i))\right.$

Definition $2 A$ rational-interval representation of a real number $x \in \mathbb{R}$ is given by a computable sequence of rational intervals, $\left\langle s_{0}, \ldots, s_{i} \ldots\right\rangle$ with $s_{i}=\left[a_{i}, b_{i}\right]$ such that:

i) $s_{i+1} \subseteq s_{i}$

ii) $\lim _{i \rightarrow \infty}\left(b_{i}-a_{i}\right)=0$

iii) $x=\bigcap_{i \in \mathbb{N}} s(i)$.

This representation has been used by several authors in the real number computability, see Lacombe, Martin-Löf, Scott, Weihrauch, [Lac59], [ML70], [Sco70], [WS81]. In many ways, it can be considered to be the general form of real number representation. Many other representations proposed in the literature differ from this one only in that they make use of a subset of the convergent sequences of rational intervals. Here are some examples:

Definition 3 a) a real number $x$ is represented by a computable Cauchy sequence of rational numbers $\left\langle a_{0}, \ldots, a_{i}, \ldots\right\rangle$ and by a computable function $q: \mathbb{N} \rightarrow \mathbb{N}$ defining the convergence rate of the Cauchy sequence, i.e.:

i) $\forall i . j . k .\left|a_{q(i)+j}-a_{q(i)+k}\right| \leq 2^{-i}$

ii) $x=\lim _{i \rightarrow \infty} a_{i}$

b) a real number $x$ is represented by a computable Cauchy sequence of rational numbers $\left\langle a_{0}, \ldots, a_{i}, \ldots\right\rangle$ having a fixed rate of convergence, i.e.:

i) $\forall i . j .\left|a_{i}-a_{i+j}\right| \leq 1 / i$

ii) $x=\lim _{i \rightarrow \infty} a_{i}$

c) corresponding to every natural number $p>1$, we have the following form of real representation: a real number $x$ is represented by a computable sequence 
of integers $\left\langle z_{0}, \ldots, z_{i}, \ldots\right\rangle$ such that:

i) $\forall i .\left|p \times z_{i}-z_{i+1}\right|<p$

ii) $x=\lim _{i \rightarrow \infty} z_{i} / p^{i}$

d) given a natural number $b>1$ a negative-digit representation with base $b$ of a real number $x$ is given by a computable sequence of integers $\left\langle z_{0}, \ldots, z_{i}, \ldots\right\rangle$, such that:

i) $\forall i \in \mathbb{N}^{+} .-b<z_{i}<b$

ii) $x=\sum_{i \in \mathbb{N}} z_{i} \times b^{-i}$

e) in the continued fraction representation a real number $x$ is represented by a computable sequence of integers $z_{0}, \ldots, z_{i} \ldots$ such that:

$$
x=\lim _{i \rightarrow \infty} z_{0}+\frac{1}{z_{1}+\frac{1}{z_{2}+\frac{1}{\vdots}}}
$$

Representations a) and b) are used in Troelstra, [TvD88] and in Bishop [Bis67] respectively. Representations a ) and b) are similar to the classical Cauchy sequence representation. Notice however that the constructive definition of a real number via a Cauchy sequence always requires the presence of a function defining the convergence rate. This convergence function can be the same for all Cauchy sequences, like in representation b), or can be specified individually for each Cauchy sequence, like in representation a). An informal justification for the necessity of introducing a function giving the convergence rate is the following: if the convergence rate of a Cauchy sequence $\left\langle a_{0}, a_{1}, \ldots\right\rangle$ with limit $x$ is unknown then it is impossible to give any approximation to $x$ after examining a finite subsequence $\left\langle a_{0}, \ldots, a_{i}\right\rangle$, in fact any real number can be the limit of a Cauchy sequence starting with $\left\langle a_{0}, \ldots, a_{i}\right\rangle$. This is of paramount importance; in fact from a constructive point of view only finite parts of an infinite sequence can be examined.

Representation c) is used in Boehm, [BCRO86]. It can be considered a variant of the Cauchy sequence representation. Here a sequence of integers is used to describe a Cauchy sequence of rational p-adic numbers. A p-adic rational number is a number that can be written in the form $m \times p^{-n}$ with $m$ and $n$ integers. For the practical purposes representation c) is convenient: the algorithms for the arithmetic operations turn out to be simpler and more efficient when representation c) is used instead of representations a) or b).

Representation d) is similar to the standard digited representation. The main difference consists in introducing negative digits. This representation has been studied in Avizienis [Avi64], Boehm [BCRO86] and Wiedmer [Wie80].

The representation e) is developed in Vuillemin [Vui88] and is similar to the standard continued fraction representation. The only difference is that in the standard continued fraction notation only natural numbers are used. In this case, however, negative integers are also used.

The representations described above do not make explicit use of intervals. However perfectly equivalent representations based on rational intervals can be 
given. Let us consider for example representation $\mathrm{b}$ ). A real number $x$ is defined by a Cauchy sequence of rational numbers $\left\langle a_{0}, \ldots, a_{i}, \ldots\right\rangle$. If we examine a finite part of the sequence $s$ we can give an estimation for $x$. From the element $a_{i}$ we know that the value of $x$ lies in the interval: $\left[a_{i}-1 / i, a_{i}+1 / i\right]$. The same information is given by the sequence of rational intervals:

$\left\langle\left[a_{1}-1, a_{1}+1\right], \ldots,\left[a_{i}-1 / i, a_{i}+1 / i\right], \ldots\right\rangle$.

Analogous considerations can be made for the other representations.

As mentioned above the representations presented in d) and in e) are modifications of the digited representation and of the standard continued fraction representation respectively. The reason for these modifications is that the standard representations are not suitable for real number computation. Using the standard representations even the most fundamental functions such as addition or multiplication are not computable.

Here is a simple example that illustrates the inadequacy of the standard decimal representation. We show that no algorithm can compute multiplication by 3. A hypothetical algorithm for this function will not be able to generate the first digit of the result when it receives as input the value $0.333 \ldots$. In this case there are two possible results, namely $1.000 \ldots$ and $0.999 \ldots$. If the algorithm generates 1 as the first digit, this must happen after the algorithm has examined a finite number of digits of the argument. Let us suppose that the first $n$ digits have been examined before generating 1 . Then the algorithm generates 1 as first digit also when it receives as input the string $0 . \overbrace{33 \ldots 3}^{n} 00 \ldots$. But this is incorrect since the exact result should then be $0 . \overbrace{99 \ldots 9}^{n} 0 \ldots$ An analogous argument can be made if the algorithm generates 0 as first digit.

Similar examples show also that the other arithmetic operations are not computable. Clearly the problem presented above is not caused by the choice of base 10 for the representation of real numbers. The same problem would arise for any other base. The introduction of negative digits, as in representation $\mathrm{d}$ ) above, is a simple way to overcome these difficulties. The standard interpretation can be extended to strings of positive and negative digits. For example the string $0 .\langle+4\rangle\langle-5\rangle\langle-3\rangle\langle+2\rangle$ represents the rational number

$$
\left(+4 \times 10^{-1}\right)+\left(-5 \times 10^{-2}\right)+\left(-3 \times 10^{-3}\right)+\left(+2 \times 10^{-4}\right)
$$

Going back to the previous example we can easily show how the introduction of negative digits solves the difficulty. The algorithm for multiplication by 3 can in fact safely generate 1 , as the first digit, after having read the first two digits of the string $0.333 \ldots$. We can easily observe that if the input becomes $0.3(-9)(-9) \ldots=0.2$ the output can become $1 .(-4) 000 \ldots=0.6$. If the input becomes $0.3999 \ldots=0.4$ the output can become $1.2000 \ldots$. A very similar consideration can be made for the continued fraction representation.

It is possible to prove that all the previous real number representations are computationally equivalent, in the sense that they characterize the same class 
of computable reals and computable real functions. The proof consists in defining effective translations between the different representations. In constructive mathematics other representations of real numbers, not computationally equivalent to the previous ones, are also considered, for example representations based on Dedekind cuts [TvD88]. In this paper we do not discuss these representations.

\section{A domain of approximations for real numbers}

In the literature there are different approaches to computability on real numbers which make use of different sorts of domains. In one of his early papers on domain theory, Scott [Sco70] suggested that a cpo consisting of intervals of the real line can be used to study computability on real numbers. Previously also Martin-Löf [ML70] constructed a similar space of approximations. A similar idea was also presented in Lacombe [Lac59]. In all these cases the real line is embedded in spaces of approximations where a notion of computability can be defined in a natural way. Many results concerning the computability theory on real numbers are given in these contexts. These spaces of approximations are particular cases of countably based continuous partial orders whose formal theory has been developed in Smyth [Smy76]. Later Weihrauch and Schreiber [WS81] developed similar ideas in the context of algebraic cpo's enriched with a notion of distance and weight. In recent work Sünderhauf [S9̈5] considers a domain of approximation for real numbers based on the notion of quasi-uniformity.

In this paper we present a construction that is similar in many respects to the ones mentioned above but has some important differences. In constructing a space of approximations a given form of real number representation is always assumed. All constructions mentioned above are based on the representation of real numbers as converging sequences of rational intervals (Definition 2). This form of representation is not appropriate for implementations of real number computation. One can see this informally, by noting that the efficiency of the computation is certainly decreased by the existence of too many approximation points (every rational interval is an approximation point), i.e., cumbersome representations.

In view of our goals we base our construction on other forms of real number representations: the integer sequence and the digit sequence representations of Definition $3 \mathrm{c}$ ) and $\mathrm{d}$ ). These forms are in fact more suitable for use in an actual implementation. A second important difference is the following: our space of approximations turns out to be a Scott-Domain. The other approaches generate instead more general forms of cpo's, which are less used in denotational semantics.

\subsection{Domain theory preliminaries}

For completeness we briefly summarise some basic definitions of domain theory. Further details can be found [AJ94]. 
Given a partial order $\langle D, \sqsubseteq\rangle$ the following notation is used. $d \sqcup d^{\prime}$ and $d \sqcap d^{\prime}$ denote the least upper bound and the greatest lower bound of the elements $d, d^{\prime} \in D$ respectively, if they exist. Given a subset $A$ of $D, \sqcup A$ and $\sqcap A$ denote respectively the least upper bound, greatest lower bound of $A$, if they exist. A subset $A$ of $D$ is directed if it is non-empty and every pair of elements of $A$ has an upper bound in $A$. Two elements $d, d^{\prime} \in D$ are consistent if they have an upper bound, we write $d \Uparrow d^{\prime}$ for the proposition that $d, d^{\prime}$ are consistent. A downward closed (upward closed) subset of $D$ is a subset $A$ such that for all $d, d^{\prime}$ in $D$ if $d \in A$ and $d^{\prime} \sqsubseteq d\left(d \sqsubseteq d^{\prime}\right)$ then $d^{\prime} \in A$. An ideal over $\langle D, \sqsubseteq\rangle$ is a directed, downward closed subset. Given $d \in D$, the downward cone (upward cone) generated by $d$ is the set $\downarrow d=\left\{d^{\prime} \mid d \sqsubseteq d^{\prime}\right\}\left(\uparrow d=\left\{d^{\prime} \mid d \sqsubseteq d^{\prime}\right\}\right)$ An ideal $I \subseteq D$ is called principal if there exist $d \in D$ s.t. $I=\downarrow d$. We write $\operatorname{Idl}\langle D, \sqsubseteq\rangle$ for the set of the ideals over the order $\langle D, \sqsubseteq\rangle$.

A complete partial order (cpo) is a partial order $\langle D, \sqsubseteq\rangle$ where there exists a least element $\perp_{D}$ and every directed subset has lub. A finite element of a cpo $\langle D, \sqsubseteq\rangle$ is an element $d \in D$ such that for any directed subset $A$ of $D$ if $d \sqsubseteq \bigsqcup A$ then there is an element $a \in A$ such that $d \sqsubseteq a$. We write $D^{\circ}$ for the set of finite elements of $\langle D, \sqsubseteq\rangle$. A cpo $\langle D, \sqsubseteq\rangle$ is algebraic if, for each $d \in D$ the set $D^{\circ} \cap \downarrow d$ is directed and its lub is $d$. If $\langle D, \sqsubseteq\rangle$ is a partial order with a least element then $\operatorname{Idl}\langle D, \sqsubseteq\rangle$ ordered by inclusion is an algebraic cpo. The finite elements of $\operatorname{Idl}\langle D, \sqsubseteq\rangle$ are the principal ideals of $\langle D, \sqsubseteq\rangle$. The order $\langle\operatorname{Idl}\langle D, \sqsubseteq\rangle, \subseteq\rangle$ is called the the ideal completion of $\langle D, \sqsubseteq\rangle$. A cpo $\langle D, \sqsubseteq\rangle$ is $\omega$-algebraic if it is algebraic and $D^{\circ}$ is countable. A cpo $\langle D, \sqsubseteq\rangle$ is consistently complete if each pair of consistent elements has a least upper bound. A Scott-domain is a consistently complete $\omega$-algebraic cpo.

A function $f: D \rightarrow D^{\prime}$ from a cpo $D$ to a cpo $D^{\prime}$ is continuous if for all directed subset $A$ of $D$ we have: $f(\bigsqcup A)=\bigsqcup_{d \in A} f(d)$. The set $\left[D \rightarrow D^{\prime}\right]$ of continuous functions from $D$ to $D^{\prime}$ is itself a cpo under the pointwise ordering. If $d, d^{\prime}$ are finite elements of $D$ and $D^{\prime}$ we write $\left(d \Rightarrow d^{\prime}\right)$ for the element of $\left[D \rightarrow D^{\prime}\right]$ defined by: $\left(d \Rightarrow d^{\prime}\right)\left(d_{0}\right)=d^{\prime}$ if $d \sqsubseteq d_{0}$ and $\left(d \Rightarrow d^{\prime}\right)\left(d_{0}\right)=\perp$ otherwise. A function of the form $\left(d \Rightarrow d^{\prime}\right)$ is called step function. If $D$ and $D^{\prime}$ are Scott-domains so is $\left[D \rightarrow D^{\prime}\right]$. Its finite elements are the lubs of finite sets of step functions. Let $D$ and $D^{\prime}$ be two Scott-domains, $D \times D^{\prime}$ denotes the set of pairs with the pointwise order. $D \times D^{\prime}$ is itself a cpo.

Cpos can be endowed with a topological structure called Scott-topology. In a cpo $D$, a subset $O$ is open w.r.t. the Scott-topology if it is upward closed and for any directed subset $A$ of $D$ if $\bigsqcup A \in O$ then $A \cap O \neq \emptyset$. If $D$ is an algebraic cpo an equivalent definition of the Scott-topology can be given as follows: a subset $O$ is open if it is union of subsets of the form $\uparrow d$ where $d$ is a finite element. A function $f: D \rightarrow D^{\prime}$ between Scott-domains is continuous (preserves the lub's of directed sets) if and only if it is continuous w.r.t. the Scott-topologies.

An effective Scott-domain is a triple $\langle D, \sqsubseteq, \epsilon\rangle$ such that $\langle D, \sqsubseteq\rangle$ is a Scottdomain and $\epsilon$ is an enumeration of the finite elements $D^{\circ}$ such that the following relations are decidable:

i) $\epsilon(n) \Uparrow \epsilon(m) \quad$ ii) $\epsilon(n)=\epsilon(m) \sqcup \epsilon\left(m^{\prime}\right)$

An element $d$ in a effective Scott-domain $\langle D, \sqsubseteq, \epsilon\rangle$ is computable if the set 
$\{n \mid \epsilon(n) \sqsubseteq d\} \subseteq \mathbb{N}$ is recursively enumerable. If $\langle D, \sqsubseteq, \epsilon\rangle$ and $\left\langle D^{\prime}, \sqsubseteq, \epsilon^{\prime}\right\rangle$ are effective Scott-domains so are $D \times D^{\prime}$ and $\left[D \rightarrow D^{\prime}\right]$, the enumeration functions $\epsilon_{\times}$and $\epsilon_{\rightarrow}$ are defined by: $\epsilon_{\times}\left(\left\langle\left\langle n_{1}, n_{1}\right\rangle\right\rangle\right)=\left\langle\epsilon\left(n_{1}\right), \epsilon\left(n_{2}\right)\right\rangle$ and $\epsilon_{\rightarrow}\left(\left\{\left|\left\langle m_{1,1}, m_{1,2}\right\rangle\right\rangle, \ldots,\left\langle\left\langle m_{n, 1}, m_{n, 2}\right\rangle\right\rangle\right\}\right)$ is equal to $\bigsqcup_{i<n}\left(m_{i, 1} \Rightarrow m_{i, 2}\right)$ if this lub exists and it is equal to $\perp$ otherwise.

\subsection{The construction of the domain $\mathrm{RD}$}

The domain of approximations defined next is called Reals Domain $(R D)$. First we present a construction of $R D$ starting with the binary negative digit notation of real numbers, according to Definition $3 \mathrm{~d}$ ) in base 2 . Later we will show that $R D$ can be obtained also by repeating the same construction starting with the Cauchy sequence notation of real numbers. Let $\left\langle s_{i}\right\rangle_{i \in \mathbb{N}}$ be a sequence of integers defining a real number $r$ according to the binary negative digit notation and let $\left\langle s_{i}\right\rangle_{i<n}$ be an initial subsequence. $\left\langle s_{i}\right\rangle_{i<n}$ gives partial information about the value $r$. Examining $\left\langle s_{i}\right\rangle_{i<n}$ we can deduce that the value $r$ is contained in an interval of real numbers. For example the sequence $\langle 2,(-1)\rangle$ is the initial notation of a number contained in the closed interval $[1,2]$. All the sequences beginning with $2,(-1)$ denote a real contained in the interval $[1,2]$ and each number in the interval $[1,2]$ can be denoted by a sequence beginning with $2,(-1)$. This observation leads to the definition of a function from finite sequences of integers to intervals in the real line. To any finite sequence $\left\langle s_{i}\right\rangle_{i<n}$ we associate the interval $[a, b]$ containing the real numbers that can be represented by sequences having as initial subsequences $\left\langle s_{i}\right\rangle_{i<n}$.

To simplify the presentation we restrict ourselves to consider just those sequences of integers that do represent real numbers or approximations of them.

Notation We write $S^{*}, S^{\omega}, S^{\infty}$ for the partial orders composed of sequences of integers and defined by:

$$
\begin{aligned}
& S^{*}=\left\{\left\langle s_{0}, \ldots s_{n-1}\right\rangle \mid n \in \mathbb{N}, s_{0} \in Z, \forall i<n-1 . s_{i+1} \in\{-1,0,1\}\right\} \\
& S^{\omega}=\left\{\left\langle s_{i}\right\rangle_{i \in \mathbb{N}} \mid \forall n \in \mathbb{N} .\left\langle s_{i}\right\rangle_{i<n} \in S^{*}\right\} \\
& S^{\infty}=S^{*} \cup S^{\omega}
\end{aligned}
$$

with order given by the prefix relation.

Note that $S^{\infty}$ is isomorphic to the ideal completion of $S^{*}$. Next we define a monotone function $\phi$ from $S^{*}$ to the partial order formed by the closed intervals of the real line with the superset ordering. In the following $R I$ will denote the partial order of closed intervals of the real line. Before giving the definition of $\phi$ we need to extend the arithmetic operations to $R I$.

Definition 4 The arithmetic operations on $R I$ are defined by:

$[a, b]+\left[a^{\prime}, b^{\prime}\right]=\left[a+a^{\prime}, b+b^{\prime}\right]$

$-[a, b]=[-b,-a]$

$[a, b] \times\left[a^{\prime}, b^{\prime}\right]=\left[\min \left\{a \times a^{\prime}, a \times b^{\prime}, b \times a^{\prime}, b \times b^{\prime}\right\}, \max \left\{a \times a^{\prime}, a \times b^{\prime}, b \times a^{\prime}, b \times b^{\prime}\right\}\right]$

$1 \div[a, b]= \begin{cases}{[-\infty,+\infty]} & \text { if } 0 \in[a, b] \\ {[1 \div b, 1 \div a]} & \text { otherwise }\end{cases}$

where with $[-\infty,+\infty]$ we denote the interval consisting of the whole real line. 
A justification for the above definition is the following: $[a, b]+\left[a^{\prime}, b^{\prime}\right]$ is the interval of values that are obtained by adding an element of $[a, b]$ to an element of $\left[a^{\prime}, b^{\prime}\right]$. It is easy to check that similar considerations are valid for the other arithmetic operations.

Notation If $a^{\prime}$ is a rational number and $[a, b]$ is a closed interval in $\mathbb{R}$, we use the abbreviation $a^{\prime}+[a, b]$ to denote the interval $\left[a^{\prime}, a^{\prime}\right]+[a, b]$. Similar abbreviations are used also for the other arithmetic operations.

Definition 5 The function $\phi: S^{*} \rightarrow R I$ is recursively defined by:

$$
\begin{aligned}
& \phi(\langle\rangle)=[-\infty,+\infty] \\
& \phi\left(\left\langle s_{0}, s_{1}, \ldots, s_{n}\right\rangle\right)=s_{0}+\phi^{\prime}\left(\left\langle s_{1}, \ldots, s_{n}\right\rangle\right) \\
& \phi^{\prime}(\langle\rangle)=[-1,1] \\
& \phi^{\prime}\left(\left\langle s_{0}, s_{1}, \ldots, s_{n}\right\rangle\right)=\left(s_{0}+\phi^{\prime}\left(\left\langle s_{1}, \ldots, s_{n}\right\rangle\right)\right) \div 2
\end{aligned}
$$

The left and the right endpoint of the interval $\phi\left(\left\langle s_{i}\right\rangle_{i<n}\right)$ denote respectively the smallest and the largest number that can be denoted by an element greater than $\left\langle s_{i}\right\rangle_{i<n}$ (under the substring order). Every number inside the interval $\phi\left(\left\langle s_{i}\right\rangle_{i<n}\right)$ can be denoted by a proper infinite superstring of $\left\langle s_{i}\right\rangle_{i<n}$.

Examples:

$\phi(\langle 0\rangle)=[-1,1], \quad \phi(\langle 0,1\rangle)=[0,1], \quad \phi(\langle 0,1,0\rangle)=[1 / 4,3 / 4]$.

It is not difficult to verify that the image of the function $\phi$ is given by the whole real line $([\infty,+\infty])$ and by the intervals having form $\left[(z-1) / 2^{n},(z+1) / 2^{n}\right]$ where $n$ is a natural and $z$ is an integer. The rational numbers in the form $z / 2^{n}$ with $n \in \mathbb{N}, z \in Z$ are called dyadic rationals. We call dyadic intervals the rational intervals having the form $[-\infty,+\infty]$ or $\left[(z-1) / 2^{n},(z+1) / 2^{n}\right]$ where $n \in \mathbb{N}$ and $z \in Z$.

Notation $D$ will indicate the set of rational dyadic numbers and $(D I, \sqsubseteq)$ will indicate the partial order of the dyadic intervals. The order relation $\sqsubseteq$ on $D I$ is the superset relation, that is $[a, b] \sqsubseteq\left[a^{\prime}, b^{\prime}\right]$ if and only if $\left[a^{\prime}, b^{\prime}\right] \subseteq[a, b]$.

A useful property of the partial order $(D I, \sqsubseteq)$ is derived from the following:

Proposition 1 The intersection of any pair of dyadic intervals is empty or reduces to a single point or is itself a dyadic interval.

Proof Let $\left[\left(z_{1}-1\right) / 2^{n_{1}},\left(z_{1}+1\right) / 2^{n_{1}}\right]$ and $\left[\left(z_{2}-1\right) / 2^{n_{2}},\left(z_{2}+1\right) / 2^{n_{2}}\right]$ be two dyadic intervals. The proof is by case analysis on the order relation existing between the limits of the two dyadic intervals. Let us consider the case where $\left(z_{1}-1\right) / 2^{n_{1}}<\left(z_{2}-1\right) / 2^{n_{2}}<\left(z_{1}+1\right) / 2^{n_{1}}<\left(z_{2}+1\right) / 2^{n_{2}}$ with $n_{1} \leq n_{2}$. Therefore $z_{2}-1<\left(z_{1}+1\right) \times 2^{n_{2}-n_{1}}<z_{2}+1$ and so: $\left(z_{1}+1\right) \times 2^{n_{2}-n_{1}}=z_{2}$. It follows that:

$\left[\left(z_{1}-1\right) / 2^{n_{1}},\left(z_{1}+1\right) / 2^{n_{1}}\right] \cap\left[\left(z_{2}-1\right) / 2^{n_{2}},\left(z_{2}+1\right) / 2^{n_{2}}\right]$

$=\left[\left(z_{2}-1\right) / 2^{n_{2}}, z_{2} / 2^{n_{2}}\right]$

$\left.\left.=\left[\left(2 \times z_{2}-1\right)-1\right) / 2^{n_{2}+1},\left(2 \times z_{2}-1\right)+1\right) / 2^{n_{2}+1}\right]$

which is a dyadic interval.

All other cases are trivial or similar to this one. 


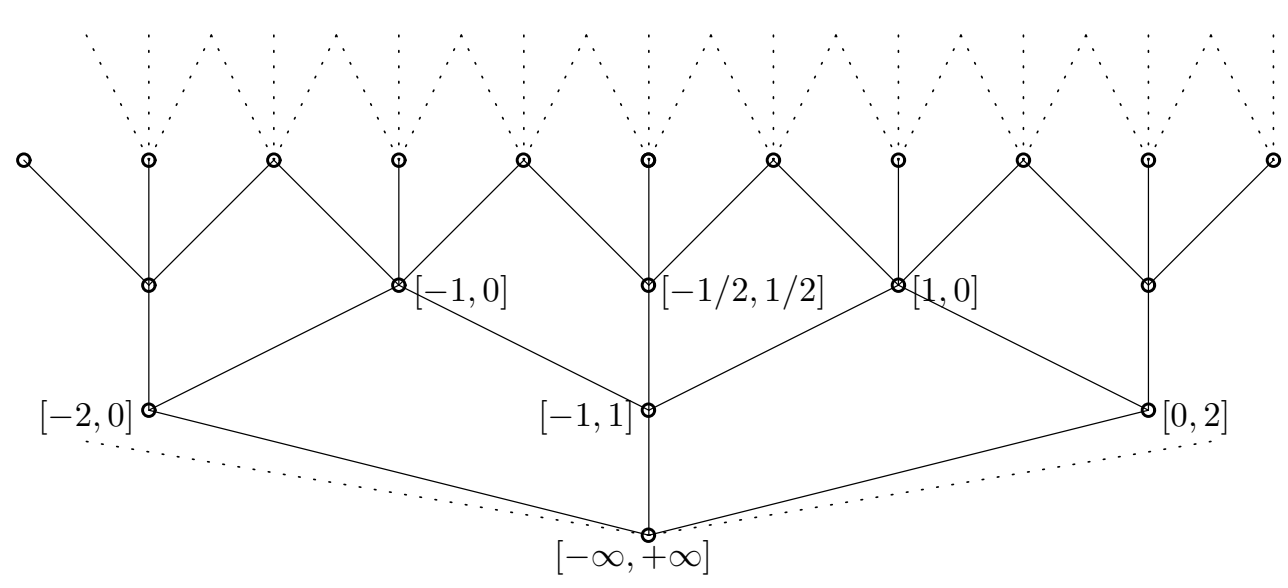

Figure 1: The diagram representing $D I$.

To extend the function $\phi$ to the set $S^{\infty}$ it is necessary to complete the partial order $D I$.

Definition 6 Let $R D$ denote the cpo obtained by the ideal completion of $(D I, \sqsubseteq)$.

Proposition $2 R D$ is a consistently complete $\omega$-algebraic cpo.

Proof Since $R D$ is the ideal completion of a countable partial order, it is $\omega$ algebraic cpo. From Proposition 1 it follows immediately that $R D$ is consistently complete.

The function $\phi$ is monotone and therefore it can be extended by continuity to a function $\bar{\phi}: S^{\infty} \rightarrow R D$.

$$
\begin{aligned}
& \bar{\phi}\left(\left\langle s_{i}\right\rangle_{i<n}\right)=\downarrow \phi\left(\left\langle s_{i}\right\rangle_{i<n}\right), \\
& \bar{\phi}\left(\left\langle s_{i}\right\rangle_{i \in N}\right)=\left\{[a, b] \mid \exists n \in \mathbb{N} . \phi\left(\left\langle s_{i}\right\rangle_{i<n}\right) \subseteq[a, b]\right\} .
\end{aligned}
$$

The domain $R D$ can be thought as composed of equivalence classes of elements $S^{\infty}$. The equivalence classes of finite elements are composed of finite sequences containing identical information about the real value they approximate (via the binary negative digit notation).

It is interesting to observe that $R D$ can be obtained also repeating the previous construction using a different notation for the real numbers. Instead of the binary negative digit notation, the Cauchy sequence notation of reals presented in Definition 3 c) (with $p=2$ ) can be considered. In this case the repetition of the previous construction leads to the definition of a different set of meaningful sequences $S_{1}^{\infty}$ but also to the definition of exactly the same domain 
$R D$ and of a function $\bar{\phi}_{1}: S_{1}^{\infty} \rightarrow R D$ that maps each element in $S_{1}^{\infty}$ to the interval of real numbers that it approximates via the Cauchy sequence notation.

$$
\begin{aligned}
& S_{1}^{*}=\left\{\left\langle s_{0}, \ldots, s_{n-1}\right\rangle|n \in \mathbb{N}, \forall i<n-1 .| 2 \times s_{i}-s_{i+1} \mid<2\right\} \\
& S^{\infty}=S_{1}^{*} \cup\left\{\left\langle s_{i}\right\rangle_{i \in \mathbb{N}} \mid \forall n \in \mathbb{N} .\left\langle s_{i}\right\rangle_{i<n} \in S_{1}^{*}\right\}
\end{aligned}
$$

and $\bar{\phi}_{1}$ is the continuous extension of the functions $\phi_{1}: S_{1}^{*} \rightarrow D I$ defined by:

$$
\begin{aligned}
& \phi_{1}(\langle\rangle)=\perp \\
& \phi_{1}\left(\left\langle s_{0}, \ldots, s_{n}\right\rangle\right)=\left[s_{n}-1, s_{n}+1\right] \div 2^{n}
\end{aligned}
$$

In an alternative presentation one can also consider all the possible sequences of integers, instead of restricting to the meaningful ones. In this case one needs to introduce the empty interval to denote "inconsistent" sequences, that is, sequences that neither approximate nor denote any real number. The empty interval will be the maximum element of the alternative domain $R D_{\top}$.

\subsection{Computability}

Here we use the effective coding function of pairs of natural numbers $\langle\langle\rangle$ and and the enumeration function for the integers $\epsilon_{Z}$ presented Definition 1.

Proposition 3 Let $\epsilon_{r}$ be the enumeration of the finite elements of $R D$ defined by:

$$
\begin{gathered}
\epsilon_{r}(0)=\perp \\
\epsilon_{r}\left(\left\langle\left\langle n_{1}, n_{2}\right\rangle\right\rangle+1\right)=\downarrow\left[\left(\epsilon_{Z}\left(n_{1}\right)-1\right) / 2^{n_{2}},\left(\epsilon_{Z}\left(n_{1}\right)+1\right) / 2^{n_{2}}\right] .
\end{gathered}
$$

$\left(R D, \sqsubseteq, \epsilon_{r}\right)$ is then an effective Scott-domain.

Proof The function $\epsilon_{r}$ clearly enumerates all the finite elements of $R D$. Moreover: $\epsilon_{r}(n) \Uparrow \epsilon_{r}\left(n^{\prime}\right)$ iff $n=0 \vee n^{\prime}=0 \vee n=\left\langle\left\langle n_{1}, n_{2}\right\rangle\right\rangle+1 \wedge n^{\prime}=\left\langle\left\langle n_{1}^{\prime}, n_{2}^{\prime}\right\rangle\right\rangle+1 \wedge$ $\left|\epsilon_{Z}\left(n_{1}\right) / 2^{n_{2}}-\epsilon_{Z}\left(n_{1}^{\prime}\right) / 2^{n_{2}^{\prime}}\right|<2^{-n_{2}}+2^{-n_{2}^{\prime}}$

it follows that the relation $\epsilon(n) \Uparrow \epsilon(m)$ is decidable. A similar argument proves the decidability of the relation $\epsilon_{r}(n)=\epsilon_{r}\left(n^{\prime}\right) \sqcup \epsilon_{r}\left(n^{\prime \prime}\right)$.

In general, given an effective Scott-domain $(D, \sqsubseteq, \epsilon)$, there might exist an alternative effective enumerations $\epsilon^{\prime}$ of the finite elements of $D$ such that $(D, \sqsubseteq, \epsilon)$ and $\left(D, \sqsubseteq, \epsilon^{\prime}\right)$ have different sets of computable elements. However the set of computable elements of $R D$ remains the same if we take a "reasonable" enumeration $\epsilon_{r}^{\prime}$ of the finite elements of $R D$, i.e. an enumeration for which the relation:

$$
\epsilon_{r}^{\prime}\left(n_{0}\right)=\downarrow\left[\left(n_{1}-n_{2}-1\right) / 2^{n_{3}},\left(n_{1}-n_{2}+1\right) / 2^{n_{3}}\right]
$$

is decidable. Note that an enumeration is reasonable if there is an effective method that given the code of a dyadic interval evaluates its limit points. It is not difficult to prove that for any reasonable enumeration $\epsilon_{r}^{\prime}$ there exist two recursive functions $f$ and $g$ s.t. for any natural $n, \epsilon_{r}(n)=\epsilon_{r}^{\prime}(f(n))$ and $\epsilon_{r}^{\prime}(n)=$ 
$\epsilon_{r}(g(n))$. elements of $D$ such that $(D, \sqsubseteq, \epsilon)$. Therefore $\epsilon_{r}$ and $\epsilon_{r}^{\prime}$ define the same set of computable elements.

Since $R D$ is an effective Scott-Domain we can apply to it the standard machinery for defining computability. In the next section we will exploit this fact to give a definition of computability for real numbers and for the functions acting on them.

\subsection{Infinite elements}

In this subsection we investigate the relation existing between the set of infinite elements of $R D$ and the real line.

First we observe the following property for the infinite elements of $R D$.

Proposition 4 For every infinite element $d \in R D$ there exists a real number $x$ such that $\bigcap_{[a, b] \in d}[a, b]=\{x\}$.

Proof For any $d \in R D$ the set $\bigcap_{[a, b] \in d}[a, b]$ cannot be empty, in fact, since $\bigcap_{[a, b] \in d}[a, b]$ is an intersection of compact closed sets it is empty if and only if there is a finite subset of intervals in $d$ whose intersection is empty, but this is in contradiction with $d$ being a directed set. Now, if $d$ is an infinite element it cannot be the case that $\{x, y\} \subseteq \bigcap_{\left[a^{\prime}, b^{\prime}\right] \in d}\left[a^{\prime}, b^{\prime}\right]$ and $x \neq y$. In fact for each pair of real numbers $x, y$ with $x \neq y$ there are just a finite number of dyadic intervals containing both $x$ and $y$, and this is in contradiction with the hypothesis of $d$ being infinite.

The relationship existing between the real line and the infinite elements of $R D$ can be clarified by means of following functions:

Definition 7 A function $q_{\mathcal{P}}: R D \rightarrow \mathcal{P}(\mathbb{R})$ is defined by:

$$
q_{\mathcal{P}}(d)=\bigcap_{[a, b] \in d}[a, b]
$$

Conversely, three functions $e, e^{-}, e^{+}: \mathbb{R} \rightarrow R D$ are defined by:

$$
\begin{aligned}
& e(x)=\{[a, b] \in D I \mid x \in(a, b)\} \\
& e^{-}(x)=\{[a, b] \in D I \mid x \in(a, b]\} \\
& e^{+}(x)=\{[a, b] \in D I \mid x \in[a, b)\}
\end{aligned}
$$

where $(a, b)$ indicates the open interval from $a$ to $b$ and $(a, b]$ and $[a, b)$ indicate the obvious part open, part closed intervals.

Proposition 5 The following statements hold:

i) for every finite element $d=\downarrow[a, b]$ in $R D, q_{\mathcal{P}}(d)=[a, b]$,

ii) for every real number $x,\{x\}=q_{\mathcal{P}} \circ e(x)=q_{\mathcal{P}} \circ e^{-}(x)=q_{\mathcal{P}} \circ e^{-}(x)$,

iii) for every non-dyadic number $x \in \mathbb{R} / D, e(x)=e^{-}(x)=e^{+}(x)$,

iv) for every dyadic number $x \in D, e(x) \sqsubset e^{-}(x), e(x) \sqsubset e^{+}(x)$ and $e^{-}(x)$ is not consistent with $e^{+}(x)$,

v) $e(\mathbb{R}) \cup e^{-}(\mathbb{R}) \cup e^{+}(\mathbb{R})$ is equal to the set of infinite elements of $R D$. 
The proof is easy.

Next we consider the function $v: S^{\omega} \rightarrow \mathbb{R}$ that associates to each meaningful sequence of integers the real number represented by it. We want to show the relation between the function $v$ and the functions $\bar{\phi}, q_{\mathcal{P}}$ and $e$ previously defined.

Definition 8 The function $v: S^{\omega} \rightarrow \mathbb{R}$ is defined by:

$$
v\left(\left\langle s_{i}\right\rangle_{i \in \mathbb{N}}\right)=\sum_{n \in N} s_{n} / 2^{n}
$$

It is not difficult to prove that:

Proposition 6 For every $s \in S^{\omega},\{v(s)\}=\left(q_{\mathcal{P}} \circ \bar{\phi}\right)(s)$

Given a dyadic number $a$ we can divide the sequences of integers representing it into three sets, the sequences ending with a series of 0 , the ones ending with a series of 1 , and the ones ending with a series of -1 ,

$$
\begin{aligned}
& \bar{a}=\left\{\left\langle s_{i}\right\rangle_{i \in \mathbb{N}} \in S^{\omega} \mid v\left(\left\langle s_{i}\right\rangle_{i \in \mathbb{N}}\right)=a, \exists j . \forall i>j . s_{i}=0\right\}, \\
& \overline{a^{-}}=\left\{\left\langle s_{i}\right\rangle_{i \in \mathbb{N}} \in S^{\omega} \mid v\left(\left\langle s_{i}\right\rangle_{i \in \mathbb{N}}\right)=a, \exists j . \forall i>j . s_{i}=1\right\}, \\
& \overline{a^{+}}=\left\{\left\langle s_{i}\right\rangle_{i \in \mathbb{N}} \in S^{\omega} \mid v\left(\left\langle s_{i}\right\rangle_{i \in \mathbb{N}}\right)=a, \exists j . \forall i>j . s_{i}=-1\right\},
\end{aligned}
$$

it is possible to prove that any sequence representing $a$ is contained in one of these sets and moreover:

$$
\forall s \in \bar{a} . \bar{\phi}(s)=e(a), \quad \forall s \in \overline{a^{+}} . \bar{\phi}(s)=e^{+}(a), \quad \forall s \in \overline{a^{-}} . \bar{\phi}(s)=e^{-}(a)
$$

We can say that the infinite elements of $R D$ are a close representation of the real line, the set of infinite elements in $R D$ looks like the real line except that each dyadic number is triplicated.

In the next section we will show how to solve the problem of multiple representations by means of a retract construction.

\section{Topological characterisations}

In this section we present some results concerning the topological relationship between the real line and the Scott-domain $R D$. These results are then generalised to function spaces. Using $R D$ we give also a definition of computable real and of computable function on reals. Topologically the domain $R D$ is much more tightly related with the real line that the domain $S^{\infty}$ whose elements were originally used to represent real numbers. This fact is true also for function spaces. As a consequence, the use of $R D$ makes it easier to prove topological properties of the computable functions on the reals.

The main topological relation considered in this section is the notion of retraction between spaces. The real line turns out to be a retract of the subspace of infinite elements of $R D$. 


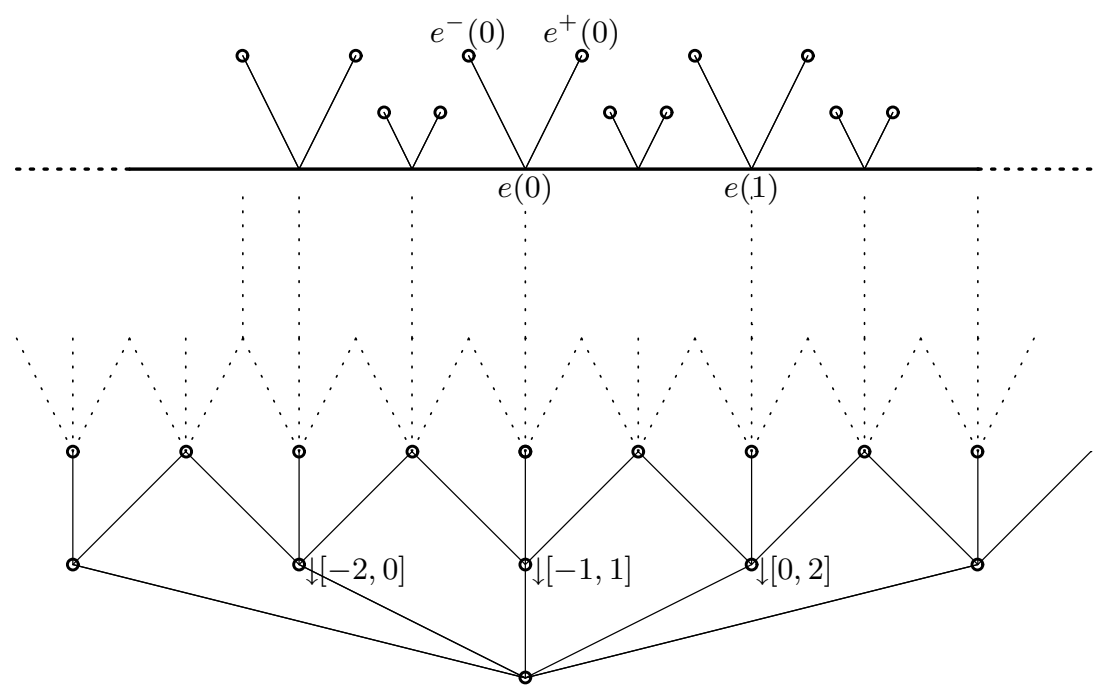

Figure 2: The diagram representing $R D$.

\subsection{Topological preliminaries}

For completeness we give here definitions of the topological notions that will we require.

Let $S$ be a subset of a topological space $T$. The subspace topology on $S$ is defined as follows: a set $O$ in $S$ is an open set in the subspace topology if and only if there is an open set $O^{\prime}$ in $T$ such that $O=S \cap O^{\prime}$

Let $Q$ be an equivalence relation on a space $T$. Let $\phi$ denote the canonical map from $T$ to $T / Q, \phi(x)=[x]$. The quotient topology on $T / Q$ is defined by: a set $O$ in $T / Q$ is open if and only if $\phi^{-1}(O)$ is open.

A space $S$ is said to be a retract of a space $T$ if there are two continuous functions $q: T \rightarrow S$ and $e: S \rightarrow T$ such that $q \circ e=I d_{S}$. In this case the following statements hold:

1) $S$ is homeomorphic to the subspace $e(S)$ of $T$

2) Let $Q$ denote the equivalence relation induced on $T$ by $q, S$ is homeomorphic to the quotient space $T / Q$.

A subbase $P$ of a space $T$ is a family of open sets of $T$ such that any other open set $O$ of $T$ can be written as a union of finite intersections of sets in $P$. Let $X$ be a set, given a family $P$ of subsets of $X$ there is a unique topology on $X$ such that $P$ forms a subbase for that topology.

Let $S$ and $T$ be two topological spaces, and $S \rightarrow T$ be set of the continuous functions from $S$ to $T$. The compact-open topology on $S \rightarrow T$, is the topology having as subbase the sets in the form $C \Rightarrow O=\{f \mid f(C) \subseteq O\}$, where $C$ is any compact set in $S$ and $O$ is any open set in $T$. See [Dug66] for a more complete 
treatment.

\subsection{The topological relation between the domain $\mathrm{RD}$ and the real line}

Let $R D^{\dagger}$ denote the subspace of $R D$ consisting of the infinite elements with the subspace Scott-topology on $R D$.

Proposition 7 The real line is a retract of $R D^{\dagger}$ via a pair of continuous functions $q: R D^{\dagger} \rightarrow \mathbb{R}$ and $e: \mathbb{R} \rightarrow R D^{\dagger}$ with

$$
\begin{gathered}
q(d)=x \text { iff } q_{\mathcal{P}}(d)=\{x\}\left(=\bigcap_{[a, b] \in d}[a, b]\right) \\
e(x)=\{[a, b] \in D I \mid x \in(a, b)\}
\end{gathered}
$$

Proof From Propositions 4 and 5 it follows that the functions $q$ and $e$ are well defined and that $q \circ e=i d_{\mathbb{R}}$. We need to prove that the two functions are continuous. We first prove the continuity of $e$. Let $x \in \mathbb{R}$ and $O$ a Scott-open set in $R D$ such that $e(x) \in O$ then there exists a finite element $\downarrow[a, b]$ such that $\downarrow[a, b] \sqsubseteq e(x)$ and $\downarrow[a, b] \in O$. It follows that $(a, b)$ is an open neighbourhood of $x$ and for any $y \in(a, b), \downarrow[a, b] \sqsubseteq e(y)$, hence $e(y) \in O$, therefore $e((a, b)) \subseteq O$.

To prove the continuity of $q$, let $d \in R D^{\dagger}$ and let $O$ be an open set containing $q(d)$. Then there exists a dyadic interval $[a, b]$ such that $q(d) \in(a, b)$ and $[a, b] \subseteq O$. It follows that $\uparrow_{R D}\left(\downarrow_{D I}[a, b]\right) \cap R D^{\dagger}$ is an open set in $R D^{\dagger}$, $d \in \uparrow \downarrow[a, b] \cap R D^{\dagger}$ and $q\left(\uparrow \downarrow[a, b] \cap R D^{\dagger}\right) \subseteq O$.

The function $q$ associates to each element of $R D^{\dagger}$ the corresponding real number. We can interpret $e$ as the function which picks a canonical representative for each real number. Using $q$ it is possible to give a definition of computable real number:

Definition 9 A real number $x$ is computable if there is a computable element $d \in R D$ such that $x=q(d)$.

It is straightforward to prove that a real number is computable according to the above definition if and only if it is representable by a computable sequence according to Definition 2. It follows that this definition coincides with other definitions of computable real number given in the literature, see Aberth [Abe80], Grzegorczyk [Grz57], Martin-Löf [ML70], Rice [Ric54], Turing [Tur37].

Using $e$ and $q$ it is possible to associate to each Scott-continuous function $f: R D \rightarrow R D$ a partial real function $\bar{f}: \mathbb{R} \rightarrow \mathbb{R}$ defined by $\bar{f}=q \circ f \circ e$, which is partial because $q$ is defined only on the infinite elements. $\bar{f}$ is the function on reals represented by $f$. We obtain in this way a new definition of computable function on real numbers.

Definition $10 A$ (partial) function $g: \mathbb{R} \rightarrow \mathbb{R}$ is computable if there exits a computable function $f: R D \rightarrow R D$ such that $g=\bar{f}$. 
The definition of computable function allows us to associate to every element $f$ in $[R D \rightarrow R D]$ a function on real numbers. If the function $f$ is not sufficiently defined, that is if $f$ maps some infinite element to a finite element, then the associated function is a partial function.

For every Scott-continuous function $f: R D \rightarrow R D$, the function $\bar{f}$ is a composition of continuous functions and therefore is continuous. In this way we obtain a new proof of a classical result in computable analysis: every computable function on real numbers is continuous w.r.t. the Euclidean topology.

In the following we extend the notion of computability to functions of several arguments and to higher order functions. We will show how the retract relation existing between $\mathbb{R}$ and $R D^{\dagger}$ can also be extended to function spaces. However the retract relation cannot be extended to second-order functional spaces. For second-order functional spaces, a set theoretical relation is stated. We do not introduce here definitions for functionals on reals having order higher than 2 . This is not a severe limitation, in fact in mathematical analysis functionals having order higher than 2 are almost never employed.

Definition 11 For each natural number n,

i) the topological space $\mathbb{F}_{n}$ is defined by:

$$
\mathbb{F}_{n}=\left\{f: \mathbb{R}^{n} \rightarrow \mathbb{R} \mid f \text { total continuous function }\right\}
$$

where $\mathbb{R}^{n}$ denotes the usual topological product of $\mathbb{R}$.

The topology on $\mathbb{F}_{n}$ is the compact-open topology.

ii) $F D_{n}$ is the effective Scott-domain of the Scott-continuous functions $\left[R D^{n} \rightarrow R D\right]$.

Remark $\mathbb{F}_{0}$ is homeomorphic to $\mathbb{R}$ and $F D_{0}$ is homeomorphic to $R D$. Moreover when the function spaces are endowed with the compact-open topology the space $(\mathbb{R} \times \mathbb{R}) \rightarrow \mathbb{R}$ is homeomorphic, via currying, to the space $\mathbb{R} \rightarrow(\mathbb{R} \rightarrow \mathbb{R})$ and in general if $T$ is a locally compact space the spaces $(S \times T) \rightarrow V$ and $S \rightarrow(T \rightarrow V)$ are homeomorphic [Dug66].

We will use the following property of the Scott-topology.

Proposition 8 For each pair of Scott-domains D, $D^{\prime}$ the Scott-topology on $\left[D \rightarrow D^{\prime}\right]$ coincides with the compact-open topology.

Proof It is easy to prove that each Scott-open subset of $\left[D \rightarrow D^{\prime}\right]$ is also open w.r.t. the compact-open topology. In fact for each finite step function $d \Rightarrow d^{\prime}$ we have $\uparrow\left(d \Rightarrow d^{\prime}\right)=\left\{f \mid f(\uparrow d) \subseteq \uparrow d^{\prime}\right\}=(\uparrow d) \Rightarrow\left(\uparrow d^{\prime}\right)$, and for each finite element $d, \uparrow d$ is both open and compact. To prove the converse implication we need to prove that given $C$, compact subset of $D$, and $O$, open subset of $D^{\prime}, C \Rightarrow 0$ is a Scott-open subset of $\left[D \rightarrow D^{\prime}\right]$. Trivially $C \Rightarrow O$ is upward closed. Let $\left\{f_{i} \mid i \in I\right\}$ be a directed set of functions such that $\bigsqcup\left\{f_{i} \mid i \in I\right\} \in C \Rightarrow O$, we need to prove that there exists $i$ s.t. $f_{i} \in C \Rightarrow O$. One easily sees that if $f_{i} \sqsubseteq f_{j}$ then $f_{i}^{-1}(O) \subseteq f_{j}^{-1}(O)$, therefore $\left\{f_{i}^{-1}(O) \mid i \in I\right\}$, with the subset relation, is a directed set of open sets. It is also easy to prove that $\left\{f_{i}^{-1}(O) \mid i \in I\right\}$ is a 
covering of $C$. By compactness of $C$ there exists $i \in I$ s.t. $C \subseteq f_{i}^{-1}(O)$, that is, $f_{i} \in C \Rightarrow O$.

We do not associate any topology to second-order functionals on reals.

Definition 12 For each n-tuple of natural numbers $\bar{m}=\left\langle m_{1}, \ldots m_{n}\right\rangle$ i) the set of functionals on reals $\mathbb{F}_{\bar{m}}$ is defined by:

$$
\left.\mathbb{F}_{\bar{m}}=\left\{f:\left(\mathbb{F}_{m_{1}} \times \ldots \times \mathbb{F}_{m_{n}}\right) \rightarrow \mathbb{R}\right) \mid f \text { total continuous function }\right\}
$$

ii) $F D_{\bar{m}}$ is the effective Scott-domain of the Scott-continuous functions $\left[\left(F D_{m_{1}} \times \ldots \times F D_{m_{n}}\right) \rightarrow R D\right]$

Observe that $F D_{\langle 0, \ldots, 0\rangle}$ is homeomorphic to $F D_{n}$.

In $R D$ not every element denotes a real number, some elements in $R D$ are just finite approximations of real numbers. Similarly not every function in $F D_{n}$ represents a function in $\mathbb{F}_{n}$. Hence, for each natural number $n$, we define a subspace $F D_{n}^{\dagger}$ of the domain $F D_{n}$. Every element in $F D_{n}^{\dagger}$ will denote an element in $\mathbb{F}_{n}$. A similar consideration is valid for the domains $F D_{\left\langle m_{1}, \ldots m_{n}\right\rangle}$. Therefore we give the definitions

$$
\begin{gathered}
F D_{n}^{\dagger}=\left\{g \in F D_{n} \mid g\left(\left(R D^{\dagger}\right)^{n}\right) \subseteq R D^{\dagger}\right\} \\
F D_{\bar{m}}^{\dagger}=\left\{g \in F D_{\bar{m}} \mid g\left(F D_{m_{1}}^{\dagger} \times \ldots \times F D_{m_{n}}^{\dagger}\right) \subseteq R D^{\dagger}\right\}
\end{gathered}
$$

The topology on $F D_{n}^{\dagger}\left(F D_{\bar{m}}^{\dagger}\right)$ is the subspace topology of Scott-topology.

Notation. In the following an n-tuple $\left\langle y_{0}, \ldots, y_{n-1}\right\rangle$ is denoted also by $\bar{y}$. If $f$ is a function on the elements of a tuple $\bar{y}, \overline{f(y)}$ denotes its pointwise application $\left\langle f\left(y_{1}\right), \ldots, f\left(y_{n-1}\right)\right\rangle$. The symbols $[a, b],\left[a^{\prime}, b^{\prime}\right],\left[a_{i}, b_{i}\right] \ldots$ are reserved for dyadic intervals. A dyadic interval $\left[a_{i}, b_{i}\right]$ is denoted also by $[a, b]_{i}$ and finally if $\overline{[a, b]}$ is an n-tuple of dyadic intervals $\prod_{i<n}[a, b]_{i}$ denotes the obvious subset of $\mathbb{R}^{n}$.

The retract relation can be extended to function spaces.

Proposition 9 For each natural number $n, \mathbb{F}_{n}$ is a retract of $F D_{n}^{\dagger}$. The pair of retract functions $q_{n}: F D_{n}^{\dagger} \rightarrow \mathbb{F}_{n}$ and $e_{n}: \mathbb{F}_{n} \rightarrow F D_{n}^{\dagger}$ are defined as follows:

$$
\begin{gathered}
q_{n}(g)(\bar{x})=q(g(\overline{e(x)})) \\
e_{n}(f)(\bar{d})=\left\{\begin{array}{l}
\perp \\
\left\{\left[a^{\prime}, b^{\prime}\right] \mid \exists \bar{\downarrow} \overline{i f, b]} \exists \bar{l}<n . d_{i}=\perp\right.
\end{array}\left(\prod_{i<n}[a, b]_{i}\right) \subseteq\left(a^{\prime}, b^{\prime}\right)\right\} \text { otherwise }
\end{gathered}
$$

Proof We prove in the order the following points:

i) $q_{n}$ is a well-defined function,

ii) $q_{n}$ is a continuous function,

iii) $e_{n}$ is a well-defined function,

iv) $e_{n}$ is a continuous function,

v) $q_{n} \circ e_{n}=i d_{\mathbb{F}_{n}}$. 
i) We need to prove that for each $g \in F D_{n}^{\dagger} q_{n}(g)$ is a totally defined continuous functions. $q_{n}(g)$ is continuous since composition of the continuous functions $g, q$ and $e . q_{n}(g)$ is totally defined since $g \in F D_{n}^{\dagger}$.

ii) To prove the continuity of $q_{n}$ it suffices to prove that for each $C$, compact subset of $\mathbb{R}^{n}$, and $O$, open subset of $\mathbb{R}$, there exists an open subset $U$ of $F D_{n}$ such that $U \cap F D_{n}^{\dagger}=q_{n}^{-1}(C \Rightarrow O)$. Since the function $\left(\prod_{i<n} e\right)$ is continuous $\left(\prod_{i<n} e\right)(C)$, is a compact subset of $R D^{n}$. Let $O^{\prime}$ be an open set in $R D$ such that $O^{\prime} \cap R D^{\dagger}=q^{-1}(O)$. Since the Scott-topology coincides with the compactopen topology, $\left(\prod_{i<n} e\right)(C) \Rightarrow O^{\prime}$ is a Scott-open subset of $F D_{n}$. In particular it is the open set we are looking for, in fact:

$\left(\left(\prod_{i<n} e\right)(C) \Rightarrow O^{\prime}\right) \cap F D_{n}^{\dagger}=$

$=\left\{g \mid g\left(\left(\prod_{i<n} e\right)(C)\right) \subseteq O^{\prime} \wedge g \in F D_{n}^{\dagger}\right\}$

$=\left\{g \mid g\left(\left(\prod_{i<n} e\right)(C)\right) \subseteq q^{-1}(O) \wedge g \in F D_{n}^{\dagger}\right\}$

$=\left\{g \mid\left(q \circ g \circ\left(\prod_{i<n} e\right)\right)(C) \subseteq O \wedge g \in F D_{n}^{\dagger}\right\}$

$=\left\{g \mid q_{n}(g) \in C \Rightarrow O\right\}=q_{n}^{-1}(C \Rightarrow O)$.

iii) From Proposition 1 it follows that $e_{n}(f)(\bar{d})$ is an ideal of $D I$, that is, an element of $R D$. It is straightforward to prove the continuity of $e_{n}(f)$. The continuity of $f$ implies that $e_{n}(f)$ maps elements of $\left(R D^{\dagger}\right)^{n}$ in $R D^{\dagger}$.

iv) $e_{n}$ is continuous since for each finite step function $\left(\overline{\downarrow[a, b]} \Rightarrow \downarrow\left[a^{\prime}, b^{\prime}\right]\right)$ in $F D_{n}, e_{n}^{-1}\left(\uparrow\left(\overline{\downarrow[a, b]} \Rightarrow \downarrow\left[a^{\prime}, b^{\prime}\right]\right) \cap F D_{n}^{\dagger}\right)$ is an open set in $\mathbb{F}_{n}$. In fact:

$e_{n}^{-1}\left(\uparrow\left(\downarrow[a, b] \Rightarrow \downarrow\left[a^{\prime}, b^{\prime}\right]\right) \cap F D_{n}^{\dagger}\right)=\left(\prod_{i<n}[a, b]_{i}\right) \Rightarrow\left(a^{\prime}, b^{\prime}\right)$ if for each $i<n$ $[a, b]_{i} \neq[-\infty,+\infty]$, and $e_{n}^{-1}\left(\uparrow\left(\overline{\downarrow[a, b]} \Rightarrow \downarrow\left[a^{\prime}, b^{\prime}\right]\right) \cap F D_{n}^{\dagger}\right)=\emptyset$ (or $\mathbb{F}_{n}$ ) otherwise.

v) The following set of equations holds:

$\left(q_{n} \circ e_{n}\right)(f)(\bar{x})=q\left(e_{n}(\underline{f)(\overline{e(x)})})\right.$

$=q\left(\left\{\left[a^{\prime}, b^{\prime}\right] \mid \exists \overline{\downarrow[a, b]} \sqsubseteq \overline{e(x)} \cdot f\left(\prod_{i<n}[a, b]_{i}\right) \subseteq\left(a^{\prime}, b^{\prime}\right)\right\}\right)$

$=q\left(\left\{\left[a^{\prime}, b^{\prime}\right] \mid \exists \overline{[a, b]} . \bar{x} \in \prod_{i<n}(a, b)_{i} \wedge f\left(\prod_{i<n}[a, b]_{i}\right) \subseteq\left(a^{\prime}, b^{\prime}\right)\right\}\right)$

$=q\left(\left\{\left[a^{\prime}, b^{\prime}\right] \mid f(\bar{x}) \in\left(a^{\prime}, b^{\prime}\right)\right\}\right) \quad$ (by continuity of $\left.f\right)$

$=q(e(f(\bar{x})))=f(\bar{x})$.

The functions $e_{n}$ and $q_{n}$ defined above are the natural generalisation of the functions $e$ and $q . q_{n}$ associates to each element of $F D_{n}^{\dagger}$ the element of $\mathbb{F}_{n}$ represented by it. $e_{n}$ chooses, for each element in $\mathbb{F}_{n}$, a canonical representation in $F D_{n}^{\dagger}$. We can also say that the function $q_{n}$ partitions $F D_{n}^{\dagger}$ into equivalence classes. All the elements contained in a single equivalence class represent the same element in $\mathbb{F}_{n}$. The function $e_{n}$ defines a canonical representation for each class.

We discuss now the problem of defining an effective method that given an element $f$ in $F D_{n}^{\dagger}$, returns the canonical representation of the equivalence class to which $f$ belongs. Such a method exists if the function $e_{n} \circ q_{n}: F D_{n}^{\dagger} \rightarrow F D_{n}^{\dagger}$ can be extended to a continuous and computable function $c_{n}: F D_{n} \rightarrow F D_{n}$.

In the following we prove that such a function $c_{n}$ exists. To do this it is convenient to introduce the notion of finite covering. 
Definition 13 Given two natural numbers $n, j$ and an element $\bar{d} \in R D^{n}$ the set of $\mathrm{j}$-coverings $\Gamma_{n}(j, \bar{d})$ of $\bar{d}$ is defined by:

$$
\Gamma_{n}(j, \bar{d})=\left\{\overline{[a, b]} \mid \forall i<n . \downarrow[a, b]_{i} \Uparrow d_{i} \wedge b_{i}-a_{i}=2^{-j}\right\}
$$

$\Gamma_{n}(j, \bar{d})$ is a set of (n-dimensional) dyadic intervals having width $2^{-j}$ such that for each $\bar{x} \in \mathbb{R}^{n}$ if $\bar{d} \sqsubseteq \overline{e(x)}$ then $\bar{x}$ is contained in the interior part of one interval in $\Gamma_{n}(j, \bar{d})$.

Lemma 10 For each $n, j, \bar{d}$ the following properties hold:

i) if for all $i<n, d_{i} \neq \perp$ then $\Gamma_{n}(j, \bar{d})$ is a finite non-empty set,

ii) for all $\overline{d^{\prime}}$ if $\bar{d} \sqsubseteq \overline{d^{\prime}}$ then $\Gamma_{n}(j, \bar{d}) \supseteq \Gamma_{n}\left(j, \overline{d^{\prime}}\right)$.

iii) for each directed set $\left\{\bar{d}_{h} \mid h \in H\right\} \quad \bar{d}=\bigsqcup_{h} \bar{d}_{h} \Rightarrow \exists h^{\prime} . \Gamma_{n}(j, \bar{d})=\Gamma_{n}\left(j, \bar{d}_{h^{\prime}}\right)$

The proof is easy.

Proposition 11 i) The function $e \circ q: R D^{\dagger} \rightarrow R D^{\dagger}$ can be extended to a continuous and computable function $c: R D \rightarrow R D$, that is $\left.c\right|_{R D^{\dagger}}=e \circ q$. $c$ is defined by:

$$
c(d)=\left\{\left[a^{\prime}, b^{\prime}\right] \mid \exists[a, b] \in d .[a, b] \subseteq\left(a^{\prime}, b^{\prime}\right)\right\}
$$

ii) For every natural number $n$ the function $e_{n} \circ q_{n}: F D_{n}^{\dagger} \rightarrow F D_{n}^{\dagger}$ can be extended to a continuous and computable function $c_{n}: F D_{n} \rightarrow F D_{n}$ defined by:

$$
c_{n}(g)(\bar{d})=\left\{\begin{array}{l}
\perp \quad \text { if } \exists i<n . d_{i}=\downarrow[-\infty,+\infty] \\
\left\{\left[a^{\prime}, b^{\prime}\right] \mid \exists j \in \mathbb{N} . \forall[a, b] \in \Gamma_{n}(j, \bar{d}) . \downarrow\left[a^{\prime}, b^{\prime}\right] \sqsubseteq c(g(\overline{\downarrow[a, b]}))\right\} \\
\text { otherwise }
\end{array}\right.
$$

Proof In order to prove the continuity and computability of $c$, let $\epsilon_{r \rightarrow r}$ be the effectively given enumeration functions of the finite elements of $R D \rightarrow R D$ (obtained from the enumeration $\epsilon_{r}$, as shown in Subsection 3.1) and let $A$ be the set of finite elements in $R D \rightarrow R D$ defined by:

$$
A=\left\{\downarrow[a, b] \Rightarrow \downarrow\left[a^{\prime}, b^{\prime}\right] \mid[a, b] \subseteq\left(a^{\prime}, b^{\prime}\right)\right\} .
$$

It is straightforward to prove that $c=\bigsqcup A$ and therefore $c$ is continuous. Moreover the set $\left\{i \mid \epsilon_{r \rightarrow r}(i) \in A\right\}$ is recursive. It follows that the set $\left\{i \mid e_{r \rightarrow r}(i) \sqsubseteq c\right\}=\left\{i \mid \exists B \subset A . B\right.$ finite, consistent and $\left.e_{r \rightarrow r}(i) \sqsubseteq \bigsqcup B\right\}$ is recursively enumerable thus $c$ is indeed computable.

In order to prove the continuity and computability of $c_{n}$ let $\epsilon_{n}$ be the effectively given enumeration of $F D_{n} \rightarrow F D_{n}$ and let $A_{n}$ be the set of finite step functions in $F D_{n} \rightarrow F D_{n}$ defined by:

$$
\begin{aligned}
A_{n}=\{ & \left(\bigsqcup_{\overline{\left[a^{\prime}, b^{\prime}\right]} \in \Gamma_{n}(j \overline{\Downarrow[a, b]})}\left(\overline{\downarrow\left[a^{\prime}, b^{\prime}\right]} \Rightarrow \downarrow\left[a^{\prime \prime}, b^{\prime \prime}\right]\right)\right) \Rightarrow\left(\overline{\downarrow[a, b]} \Rightarrow \downarrow\left[a^{\prime \prime \prime}, b^{\prime \prime \prime}\right]\right) \mid \\
& \left.j \in \mathbb{N} \wedge \overline{[a, b]} \in(D I \backslash\{\perp\})^{n} \wedge\left[a^{\prime \prime}, b^{\prime \prime}\right] \subseteq\left(a^{\prime \prime \prime}, b^{\prime \prime \prime}\right)\right\} .
\end{aligned}
$$

It is not difficult to show that $c_{n}=\bigsqcup A_{n}$, hence the function $c_{n}$ is continuous. Moreover the set $\left\{i \mid \epsilon_{n}(i) \in A_{n}\right\}$ is recursive and therefore $c_{n}$ is computable.

It is easy to prove that $\left.c\right|_{R D^{\dagger}}=e \circ q$. In order to prove the equality $\left.c_{n}\right|_{F D_{n}^{\dagger}}=e_{n} \circ q_{n}$, we make use of the following lemma 
Lemma 12 Let $g \in F D_{n}, \overline{[a, b]} \in(D I \backslash\{\perp\})^{n}$ and $\left[a^{\prime}, b^{\prime}\right] \in D I$ then $g\left(\prod_{i<n} e\left([a, b]_{i}\right)\right) \subseteq \uparrow \downarrow\left[a^{\prime}, b^{\prime}\right]$ if and only if there exists $j$ s.t. for each $\overline{\left[a^{\prime \prime}, b^{\prime \prime}\right]} \in \Gamma_{n}(j, \overline{\downarrow[a, b]})$ we have $\downarrow\left[a^{\prime}, b^{\prime}\right] \sqsubseteq g\left(\overline{\downarrow\left[a^{\prime \prime}, b^{\prime \prime}\right]}\right)$

\section{Proof of the lemma}

$(\Leftarrow) \forall \overline{\left[a^{\prime \prime}, b^{\prime \prime}\right]} \in \Gamma_{n}(j, \overline{\downarrow[a, b]}) . \downarrow\left[a^{\prime}, b^{\prime}\right] \sqsubseteq g\left(\overline{\downarrow\left[a^{\prime \prime}, b^{\prime \prime}\right]}\right)$ implies

$\forall \bar{d} \in\left(R D^{\dagger}\right)^{n} \cdot(\bar{d} \Uparrow \bar{\downarrow}[a, b]) \Rightarrow\left(\downarrow\left[a^{\prime}, b^{\prime}\right] \sqsubseteq g(\bar{d})\right)$ which implies

$\forall \bar{x} \in \overline{[a, b]} . \downarrow\left[a^{\prime}, b^{\prime}\right] \sqsubseteq g(\overline{e(x)})$ which implies

$g\left(\prod_{i<n} e\left([a, b]_{i}\right)\right) \subseteq \uparrow \downarrow\left[a^{\prime}, b^{\prime}\right]$.

$(\Rightarrow)$ Suppose for contradiction that for any $j$ there exists $\overline{\left[a^{\prime \prime}, b^{\prime \prime}\right]} \in \Gamma_{n}(j, \overline{\downarrow[a, b]})$ such that $\downarrow\left[a^{\prime}, b^{\prime}\right] \nsubseteq g\left(\overline{\downarrow\left[a^{\prime \prime}, b^{\prime \prime}\right]}\right)$. Since for all $j, \Gamma_{n}(j, \overline{\downarrow[a, b]})$ is finite, by König's lemma it is possible to find a chain $\left\langle{\overline{\left[a^{\prime \prime}, b^{\prime \prime}\right]}}_{h}\right\rangle_{h \in N}$ such that for all $h$, $\overline{\left[a^{\prime \prime}, b^{\prime \prime}\right]_{h}} \in \Gamma_{h}(\overline{\downarrow[a, b]})$ and $\downarrow\left[a^{\prime}, b^{\prime}\right] \nsubseteq g\left(\overline{\llbracket\left[a^{\prime \prime}, b^{\prime \prime}\right]_{h}}\right)$. Since for all natural $h$, . $\overline{\downarrow\left[a^{\prime \prime}, b^{\prime \prime}\right]_{h}} \Uparrow \overline{\downarrow[a, b]}$ it follows that $\left(\bigsqcup_{h} \overline{\downarrow\left[a^{\prime \prime}, b^{\prime \prime}\right]_{h}}\right) \Uparrow \overline{\downarrow[a, b]}$, we have also that

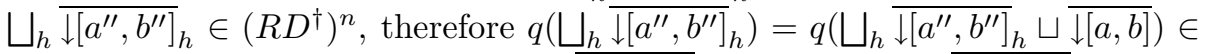

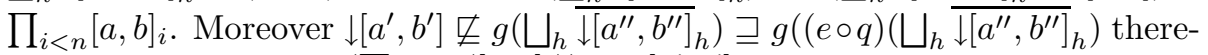
fore we can conclude $g\left(\prod_{i<n} e\left([a, b]_{i}\right)\right) \nsubseteq \uparrow \uparrow\left[a^{\prime}, b^{\prime}\right]$ which contradicts the assumption.

Now let $g \in F D_{n}^{\dagger}$, and $\bar{d} \in(R D \backslash\{\perp\})^{n}$, the following equalities hold:

$\left(e_{n} \circ q_{n}\right)(g)(\bar{d})$

$=\left\{\left[a^{\prime}, b^{\prime}\right] \mid \exists \overline{\llbracket[a, b]} \sqsubseteq \bar{d} \cdot q_{n}(g)\left(\prod_{i<n}[a, b]_{i}\right) \subseteq\left(a^{\prime}, b^{\prime}\right)\right\}$

$=\left\{\left[a^{\prime}, b^{\prime}\right] \mid \exists \overline{\llbracket[a, b]} \sqsubseteq \bar{d} \cdot q\left(g\left(\prod_{i<n} e\left([a, b]_{i}\right)\right)\right) \subseteq\left(a^{\prime}, b^{\prime}\right)\right\}$

$=\left\{\left[a^{\prime}, b^{\prime}\right] \mid \exists \overline{\llbracket[a, b]} \sqsubseteq \bar{d} \cdot c\left(g\left(\prod_{i<n} e\left([a, b]_{i}\right)\right)\right) \subseteq \uparrow \downarrow\left[a^{\prime}, b^{\prime}\right]\right\}$ (since $q(d) \in\left(a^{\prime}, b^{\prime}\right)$ iff $\left.\downarrow\left[a^{\prime}, b^{\prime}\right] \sqsubseteq c(d)\right)$

$=\left\{\left[a^{\prime}, b^{\prime}\right] \mid \exists \overline{\downarrow[a, b]} \sqsubseteq \bar{d} . \exists j \in \mathbb{N} . \forall\left[\overline{\left[a^{\prime \prime}, b^{\prime \prime}\right]} \in \Gamma_{n}(j, \overline{\downarrow[a, b]}) .\left[a^{\prime}, b^{\prime}\right] \in c\left(g\left(\overline{\downarrow\left[a^{\prime \prime}, b^{\prime \prime}\right]}\right)\right)\right\}\right.$ (by Lemma 12)

$=\left\{\left[a^{\prime}, b^{\prime}\right] \mid \exists j \in \mathbb{N} . \forall \overline{\left[a^{\prime \prime}, b^{\prime \prime}\right]} \in \Gamma_{n}(j, \bar{d}) \cdot\left[a^{\prime}, b^{\prime}\right] \in c\left(g\left(\overline{\downarrow\left[a^{\prime \prime}, b^{\prime \prime}\right]}\right)\right)\right\}$

$=c_{n}(g)(\bar{d})$ (by Lemma 10 iii) )

\subsection{Second Order Functionals}

Using the retract constructions for the first-order functions it is possible to associate to each second order functional in $F D_{\bar{m}}^{\dagger}$ the functional on the reals represented by it.

Definition 14 For every type tuple of natural numbers $\bar{m}$ let $q_{\bar{m}}$ be the function from $F D_{\bar{m}}^{\dagger}$ to $\mathbb{F}_{\bar{m}}$ defined by:

$$
q_{\bar{m}}(G)\left(f_{1}, \ldots, f_{n}\right)=q\left(G\left(e_{m_{1}}\left(f_{1}\right), \ldots, e_{m_{n}}\left(f_{n}\right)\right)\right)
$$

It is immediate that $q_{\bar{m}}$ is a well defined set theoretic function. A definition of computability for functionals follows.

Definition $15 A$ functional on real numbers $F \in \mathbb{F}_{\bar{m}}$ is computable if there exists a computable element $G \in F D_{\bar{m}}$ such that $F=q_{\bar{m}}(G)$. 
From the above a very interesting property of computable functionals follows.

Theorem 13 Every computable functional on reals is continuous w.r.t. the compact-open topology on function spaces.

This is a useful criterion for determining the non computability of functionals. Starting from this result we can easily prove that the following functionals are not computable.

1. Derivative.

$$
D(f, y)=\frac{d f}{d x}(y)
$$

2. The functional that given a function $f$ and an interval $[a, b]$ yields the minimum point $x$ in $[a, b]$ where the value $\mathrm{f}(\mathrm{x})$ is minimum.

$$
M(f, a, b)=\min \{x \mid x \in[a, b] \wedge \forall y \in[a, b] . f(x) \leq f(y)\}
$$

3. The functional that given a function $f$ and an interval $[a, b]$ yields the minimum point $x$ in $[a, b]$ where the value $f(x)$ is zero and is equal to $b$ if such a value does not exist.

$$
Z(f, a, b)=\min \{x \mid x \in[a, b], f(x)=0 \vee x=b\}
$$

In fact we can prove that none of the above functionals is continuous. For each differentiable function $f$ and for each neighbourhood $U$ of $f$ there exists $\epsilon>0$ such that the function $f_{\epsilon}(x)=f(x)+\epsilon \sin \frac{x}{\epsilon}$ belongs to $U$, moreover for each $\epsilon$ $D\left(f_{\epsilon}, 0\right)=D(f, 0)+1$, it follows that the functional $D$ is not continuous. The functional $M$ is not continuous on the function sin and interval $[-\pi, 2 \pi]$, in fact $M(\sin ,-\pi, 2 \pi)=-\pi / 2$ but for each neighbourhood $U$ of sin there exists $\epsilon>0$ such that the function $f_{\epsilon}^{\prime}(x)=\sin x-\epsilon x$ is contained in $U$ and $M\left(f_{\epsilon}^{\prime},-\pi, 2 \pi\right)=$ $\arccos \epsilon+\pi \simeq 3 / 2 \pi$. $Z$ is not continuous on the constant functions $f_{0}(x)=0$ and on the interval $[0,1]$. In fact $Z\left(f_{0}, 0,1\right)=0$ and if we defined $f^{\prime \prime}$ by $f_{\epsilon}^{\prime \prime}(x)=\epsilon-\epsilon x$ we would have that $\forall \epsilon>0 . Z\left(f_{\epsilon}^{\prime \prime}, 0,1\right)=1$.

The non-computability of the above functionals has been already proved in the literature, see [Bee85]. Such proofs consist in showing that these functionals when applied to computable functions can yield non-computable real numbers. Using Theorem 13 we have an easier proof technique. Independently and using a different approach Weihrauch [Wei95] has proved a result equivalent to Theorem 13.

It is worthwhile to notice that every functional in $F D_{m}^{\dagger}$ sends equivalent representations of the same function on reals into equivalent representations of the same real, more precisely:

Proposition 14 For each n-tuple $\bar{m}$, functional $G \in F D_{\bar{m}}^{\dagger}$ and functions $g_{1}, g_{1}^{\prime} \in F D_{m_{1}}^{\dagger}, \ldots, g_{n}, g_{n}^{\prime} \in F D_{m_{n}}^{\dagger}$ if $q_{m_{1}}\left(g_{1}\right)=q_{m_{1}}\left(g_{1}^{\prime}\right), \ldots$ and $q_{m_{n}}\left(g_{n}\right)=$ $q_{m_{n}}\left(g_{n}^{\prime}\right)$ then $q\left(G\left(g_{1}, \ldots, g_{n}\right)\right)=q\left(G\left(g_{1}^{\prime}, \ldots, g_{n}^{\prime}\right)\right)$. 
Proof It is not difficult to prove that for each $g, g^{\prime} \in F D_{n}^{\dagger}$ if $q_{n}(g)=q_{n}\left(g^{\prime}\right)$ then the greatest lower bounds of $g$ and $g^{\prime}, g \sqcap g^{\prime}$, (which exists since $F D_{n}$ is a Scott-domain) is contained in $F D_{n}^{\dagger}$. It follows that $G\left(g_{1}, \ldots, g_{n}\right) \sqsupseteq G\left(g_{1} \sqcap g_{1}^{\prime}, \ldots, g_{n} \sqcap g_{n}^{\prime}\right) \in R D^{\dagger}$ and since the only elements in $R D^{\dagger}$ that are order related are the different representations of the same real number we have: $q\left(G\left(g_{1}, \ldots, g_{n}\right)\right)=q\left(G\left(g_{1} \sqcap g_{1}^{\prime}, \ldots, g_{n} \sqcap g_{n}^{\prime}\right)\right)=q\left(G\left(g_{1}^{\prime}, \ldots, g_{n}^{\prime}\right)\right)$.

This result makes less harmful the existence, in the Scott-domains we use, of multiple representations for real numbers and for real functions.

From Proposition 14 follows that the function $q_{\bar{m}}$ can be defined by: $q_{\bar{m}}(G)=F$ if for every $g_{1} \in F D_{m_{1}}^{\dagger}, \ldots g_{n} \in F D_{m_{n}}^{\dagger}$ we have: $q\left(G\left(g_{1}, \ldots g_{n}\right)\right)=F\left(q_{m_{1}}\left(g_{1}\right), \ldots, q_{m_{n}}\left(g_{n}\right)\right)$.

Since functions can be seen as particular cases of functionals, the previous results hold also for the first-order case.

The retract construction cannot be extended to functional spaces. In particular we will prove that the function $q_{\langle 1\rangle}$ cannot be a component of a retraction pair.

Proposition 15 There is no topology on $\mathbb{F}_{\langle 1\rangle}=(\mathbb{R} \rightarrow \mathbb{R}) \rightarrow \mathbb{R}$ and no continuous function $e_{\langle 1\rangle}: \mathbb{F}_{\langle 1\rangle} \rightarrow F D_{\langle 1\rangle}^{\dagger}$ s.t. the functions $e_{\langle 1\rangle}$ and $q_{\langle 1\rangle}$ form a retraction between topological spaces.

Proof Suppose for contradiction that there exist a topology on $\mathbb{F}_{\langle 1\rangle}$ and a function $e_{\langle 1\rangle}$ making the pair $q_{\langle 1\rangle}, e_{\langle 1\rangle}$ a retraction. Let $F_{0}$ be the constant functional $\lambda f .0 \in \mathbb{F}_{\langle 1\rangle}$, let $G_{0}=e_{\langle 1\rangle}\left(F_{0}\right)$ and for each $n \in \mathbb{N}^{+}$let $g_{n} \in F D_{1}$ be the finite function defined by:

$$
g_{n}(\downarrow[a, b])= \begin{cases}\downarrow\left[-2^{-n}, 2^{-n}\right] & \text { if }-n \leq a<b \leq n \\ \perp & \text { otherwise. }\end{cases}
$$

Clearly $\bigsqcup_{n} g_{n} \in F D_{1}^{\dagger}$ so $q\left(G_{0}\left(\bigsqcup_{n} g_{n}\right)\right)=0$. By the continuity of $G_{0}$ there exists a positive natural number $l$ such that $\downarrow[-1,1] \sqsubseteq G_{0}\left(g_{l}\right)$, that is $\left(g_{l} \Rightarrow \downarrow[-1,1]\right) \sqsubseteq$ $G_{0}$. Let $O$ be the open set $O=\uparrow\left(g_{l} \Rightarrow \downarrow[-1,1]\right)$. We derive a contradiction from the set $\left(e_{\langle 1\rangle} \circ q_{\langle 1\rangle}\right)^{-1}(O)$ being open. Let $G \in F D_{\langle 1\rangle}$ be the function defined by:

$$
G(g)= \begin{cases}e(0) & \text { if } g(e(l+1)) \neq \perp \\ \perp & \text { otherwise }\end{cases}
$$

Since $q_{\langle 1\rangle}\left(G_{0}\right)=q_{\langle 1\rangle}(G)=F_{0}$ and $G_{0} \in O \subseteq q_{\langle 1\rangle}^{-1} \circ e_{\langle 1\rangle}^{-1}(O)$ it follows that $G \in q_{\langle 1\rangle}^{-1} \circ e_{\langle 1\rangle}^{-1}(O)$. So there exists a finite element $G^{\prime}$ such that: $G^{\prime} \sqsubseteq G$ and $G^{\prime} \in q_{\langle 1\rangle}^{-1} \circ e_{\langle 1\rangle}^{-1}(O) . G^{\prime}$ can be written in the form $G^{\prime}=\bigsqcup_{i<n}\left(g^{\prime}{ }_{i} \Rightarrow\left[a_{i}, b_{i}\right]\right)$, with $n \in \mathbb{N}$ and for each $i<n, g^{\prime}{ }_{i}$ is a finite function and $\left[a_{i}, b_{i}\right] \neq \perp$. Let $h \in \mathbb{N}, S_{h} \in F D_{1}^{\dagger}$ and $G_{h, l} \in F D_{\langle 1\rangle}^{\dagger}$ be defined by:

$$
h=\max \left\{b \mid \exists[a, b] . i<n \cdot[a, b] \in g^{\prime}{ }_{i}(e(l+1)) \wedge b \neq+\infty\right\}
$$




$$
\begin{gathered}
S_{h}(\downarrow[a, b])= \begin{cases}e(0) & \text { if } b \leq h \\
\downarrow[a-h, b-h] & \text { otherwise. }\end{cases} \\
G_{h, l}(g)=\left(S_{h} \circ g \circ e\right)(l+1)
\end{gathered}
$$

Notice that:

$$
\begin{gathered}
q_{1}\left(S_{h}\right)(x)= \begin{cases}0 & \text { if } x \leq h \\
x-h & \text { if } x>h .\end{cases} \\
q_{\langle 1\rangle}\left(G_{h, l}\right)(f)= \begin{cases}0 & \text { if } f(l+1) \leq h \\
f(l+1)-h & \text { if } f(l+1)>h .\end{cases}
\end{gathered}
$$

Since $G^{\prime} \sqsubseteq G$ and $\forall i<n \cdot G^{\prime}\left(g^{\prime}{ }_{i}\right) \neq \perp$ then $\forall i<n \cdot g^{\prime}{ }_{i}(e(l+1)) \neq \perp$.

Since $\forall i<n . G_{h, l}\left(g^{\prime}{ }_{i}\right)=S_{h}\left(g^{\prime}{ }_{i}(e(l+1))\right)=e(0)=G\left(g^{\prime}{ }_{i}\right) \sqsupseteq G^{\prime}\left(g^{\prime}{ }_{i}\right)$ we have that $G^{\prime} \sqsubseteq G_{h, l}$, therefore $G_{h, l} \in q_{\langle 1\rangle}^{-1} \circ e_{\langle 1\rangle}^{-1}(O)$. Now the contradiction arises from the fact that $G_{h, l}$ belongs to $q_{\langle 1\rangle}^{-1} \circ e_{\langle 1\rangle}^{-1}(O)$ but its behaviour on the elements of $F D^{\dagger}$ is inconsistent with the one of the step function $g_{l} \Rightarrow \downarrow[-1,1]$. In fact let $G^{\prime \prime}=e_{\langle 1\rangle} \circ q_{\langle 1\rangle}\left(G_{h, l}\right)$ we have that $G^{\prime \prime} \in O$ and $q_{\langle 1\rangle}\left(G^{\prime \prime}\right)=q_{\langle 1\rangle}\left(G_{h, l}\right)$, and let $f_{h, l}: \mathbb{R} \rightarrow \mathbb{R}$ be defined by:

$$
f_{h, l}(x)= \begin{cases}0 & \text { if } x \leq l \\ (h+2) \times(x-l) & \text { otherwise. }\end{cases}
$$

We have that: $q_{\langle 1\rangle}\left(G_{h, l}\right)\left(f_{h, l}\right)=q\left(G_{h, l}\left(e_{1}\left(f_{h, l}\right)\right)\right)=q\left(S_{h}\left(e_{1}(f)(e(l+1))\right)\right)$ $=q\left(S_{h}(e(h+2))\right)=2$.

On the other hand: $q_{\langle 1\rangle}\left(G_{h, l}\right)\left(f_{h, l}\right)=q_{\langle 1\rangle}\left(G^{\prime \prime}\right)\left(f_{h, l}\right)=q\left(G^{\prime \prime}\left(e_{1}\left(f_{h, l}\right)\right)\right)$ but $G^{\prime \prime}\left(e_{1}\left(f_{h, l}\right)\right) \sqsupseteq\left(g_{l} \Rightarrow \downarrow[-1,1]\right)\left(e_{1}\left(f_{h, l}\right)\right) \sqsupseteq\left(g_{l} \Rightarrow \downarrow[-1,1]\right)\left(g_{l}\right)=\downarrow[-1,1]$. Since $2 \notin[-1,1]$ we have a contradiction.

However it is possible to give a set theoretic result and prove that each functional in $\mathbb{F}_{\bar{m}}$ is the in the range of $q_{\bar{m}}$. To prove this we need to introduce the notion of $j$-approximation.

Definition 16 Given two natural numbers $n, j$ and an element $g \in F D_{n}$ the set of elements $\Delta_{n}(j, g)$ in $\mathbb{F}_{n} j$-approximated by $g$ is defined as follows:

$$
\begin{gathered}
\Delta_{0}(j, d)=\left\{x \mid \forall[a, b] \in d . x \in\left(a-2^{-j}, b+2^{-j}\right)\right\} \\
\Delta_{n}(j, g)=\left\{f \mid \begin{array}{rl}
\forall \overline{[a, b]} \in(D I)^{n} \cdot \forall\left[a^{\prime}, b^{\prime}\right] \in g(\overline{\downarrow[a, b]}) \cdot \forall \bar{x} \in \mathbb{R}^{n} . \\
& \forall i<n . x_{i} \in\left[a_{i}+2^{-j}, b_{i}-2^{-j}\right] \wedge-2^{j}<a_{i} \wedge b_{i}<2^{j} \\
\left.\Rightarrow f(\bar{x}) \in\left(a-2^{-j}, b+2^{-j}\right)\right\}
\end{array}\right.
\end{gathered}
$$

Informally, $\Delta_{n}(j, g)$ contains all the functions in $\mathbb{F}_{n}$ approximated by $g$ up to a tolerance of $2^{-j}$. We need to introduce the notion of $j$-approximation because some elements $g$ in $F D_{n}$ do not approximate any continuous functions on reals, that is the set $\uparrow g \cap F D_{n}^{\dagger}$ is empty. For an example consider: $g_{\text {step }}=(\downarrow[-1,0] \Rightarrow \downarrow[-2,-1]) \sqcup(\downarrow[0,1] \Rightarrow \downarrow[1,2])$. 
Lemma 16 For each $n, j \in \mathbb{N}$ and $g \in F D_{n}$ :

i) $\Delta_{n}(j, g)$ is an open set and $\Delta_{n}(j, g) \neq \emptyset$

ii) $\forall g^{\prime} \cdot g \sqsubseteq g^{\prime} \Rightarrow \Delta_{n}\left(j, g^{\prime}\right) \subseteq \Delta_{n}(j, g)$

iii) for each directed set $\left\{g_{h} \mid h \in H\right\} \quad g=\bigsqcup_{h} g_{h} \Rightarrow \exists h^{\prime} . \Delta_{n}(j, g)=\Delta_{n}\left(j, g_{h^{\prime}}\right)$

iv) If $g \in F D_{n}^{\dagger}$ then $q_{n}(g) \in \Delta_{n}(j, g)$ and for each open set $O$ containing $q_{n}(g)$ there exists $j^{\prime}$ s.t. $\Delta_{n}\left(j^{\prime}, g\right) \subseteq O$, in other words $\left\{\Delta_{n}(j, g) \mid j \in \mathbb{N}\right\}$ is a system of neighbourhoods for $q_{n}(g)$.

The proof is straightforward.

Proposition 17 For every $n$-tuple $\bar{m}$ of natural numbers the function $e_{\bar{m}}: \mathbb{F} \bar{m} \rightarrow F D_{m}^{\dagger}$ defined by:

$$
e_{\bar{m}}(F)\left(g_{1}, \ldots, g_{n}\right)=\left\{[a, b] \mid \exists j . F\left(\Delta_{m_{1}}\left(j, g_{1}\right), \ldots, \Delta_{m_{n}}\left(j, g_{n}\right)\right) \subseteq(a, b)\right\}
$$

is well-defined and $q_{\bar{m}} \circ e_{\bar{m}}=i d_{F_{\bar{m}}}$.

Proof From points i), ii), iii) of Lemma 16 it follows that, for each functional $F \in \mathbb{F}_{\bar{m}}, e_{\bar{m}}(F)$ is a well defined function in $F D_{\bar{m}}$.

Let $g_{1} \in F D_{m_{1}}^{\dagger}, \ldots, g_{n} \in F D_{m_{n}}^{\dagger}$, we prove that: $e_{\bar{m}}(F)\left(g_{1}, \ldots g_{n}\right)=e\left(F\left(q_{m_{1}}\left(g_{1}\right), \ldots, q_{m_{n}}\left(g_{n}\right)\right)\right)$.

The following chain of equivalences holds:

$[a, b] \in e\left(F\left(q_{m_{1}}\left(g_{1}\right), \ldots, q_{m_{n}}\left(g_{n}\right)\right)\right) \Leftrightarrow$

$F\left(q_{m_{1}}\left(g_{1}\right), \ldots, q_{m_{n}}\left(g_{n}\right)\right) \in(a, b) \Leftrightarrow$

$\exists U_{1} \ldots U_{n} . U_{i}$ neighbourhood of $q_{m_{i}}\left(g_{i}\right)$ s.t. $F\left(U_{1}, \ldots, U_{n}\right) \subseteq(a, b) \Leftrightarrow$

$\exists j . F\left(\Delta_{m_{1}}\left(j, g_{1}\right), \ldots, \Delta_{m_{n}}\left(j, g_{n}\right)\right) \subseteq(a, b) \Leftrightarrow$

$[a, b] \in e_{\bar{m}}(F)\left(g_{1}, \ldots, g_{n}\right)$.

It follows that $e_{\bar{m}}(F) \in F D_{\bar{m}}^{\dagger}$ and for every $f_{1} \in \mathbb{F}_{m_{1}}, \ldots, f_{n} \in \mathbb{F}_{m_{n}}$ we have: $\left(q_{\bar{m}} \circ e_{\bar{m}}\right)(F)\left(f_{1}, \ldots, f_{n}\right)=q\left(e_{\bar{m}}(F)\left(e_{m_{1}}\left(f_{1}\right), \ldots, e_{m_{n}}\left(f_{n}\right)\right)\right)=$

$(q \circ e)\left(F\left(\left(q_{m_{1}} \circ e_{m_{1}}\right)\left(f_{1}\right), \ldots,\left(q_{m_{n}} \circ e_{m_{n}}\right)\left(f_{n}\right)\right)\right)=F\left(f_{m_{1}}, \ldots, f_{m_{n}}\right)$.

Therefore $q_{\bar{m}} \circ e_{\bar{m}}=i d_{F_{\bar{m}}}$.

Also in this case $e_{\bar{m}}$ chooses a canonical representation for each continuous functional on $\mathbb{R}$. The fact that $q_{\bar{m}} \circ e_{\bar{m}}(F)=i d_{F_{\bar{m}}}$ implies that all continuous functionals on $\mathbb{R}$ can be represented by an appropriate functional on $R D$. By Propositions 15 there is no topology on $\mathbb{F}_{\bar{m}}$ making both functions $q_{\bar{m}}$ and $e_{\bar{m}}$ continuous.

\subsection{Partial functions}

For every natural number $n$ it is possible to extend the interpretation functions $q_{n}$ to the whole space $F D_{n}$. An element in $F D_{n}$ not contained in $F D_{n}^{\dagger}$ denotes a partial function on $\mathbb{R}$. A similar extension can be applied for any tuple $\bar{m}$ to the functions $q_{\bar{m}}$. In the following we give a topological property for the partial real functions obtained in this way. 
Definition 17 For natural number $n$ and for each n-tuple of natural numbers $\bar{m}$ let $\mathbb{F}_{n}^{p}$ and $\mathbb{F}_{\bar{m}}^{p}$ denote the set of partial functions $\mathbb{R}^{n} \rightarrow \mathbb{R}$ and $\left(\mathbb{F}_{m_{1}} \times \ldots \times \mathbb{F}_{m_{n}}\right) \rightarrow \mathbb{R}$ respectively.

The functions $p: R D \rightarrow \mathbb{R}, p_{n}: F D_{n} \rightarrow \mathbb{F}_{n}^{p}$ and $p_{\bar{m}}: F D_{\bar{m}} \rightarrow \mathbb{F}_{\bar{m}}^{p}$ are defined by:

$$
\begin{gathered}
p(d)= \begin{cases}q(d) & \text { if } d \in R D^{\dagger} \\
\text { undefined } & \text { otherwise }\end{cases} \\
p_{n}(g)\left(x_{1}, \ldots, x_{n}\right)=p\left(g\left(e\left(x_{1}\right), \ldots, e\left(x_{n}\right)\right)\right) \\
p_{\bar{m}}(G)\left(f_{1}, \ldots, f_{n}\right)=p\left(G\left(e_{m_{1}}\left(f_{1}\right), \ldots, e_{m_{n}}\left(f_{n}\right)\right)\right)
\end{gathered}
$$

Proposition 18 For each natural number $n$ and for each n-tuple $\bar{m}$ of natural number,

i) $\left.p\right|_{R D^{\dagger}}=\left.q \quad p_{n}\right|_{F D_{n}^{\dagger}}=\left.q_{n} \quad p_{\bar{m}}\right|_{F D_{\bar{m}}^{\dagger}}=q_{\bar{m}}$

ii) for each $g \in F D_{n}\left(G \in F D_{\bar{m}}\right)$, the partial function $p_{n}(g)\left(p_{\bar{m}}(G)\right)$ is continuous on its domain of definition and this domain is a countable intersection of open sets.

Proof Point i) is obvious. We prove point ii) just for second-order functions. The proof for first-order functions follows the same pattern. Since the function $q$ is continuous $p$ is continuous on its domain of definition. Let $G$ be in $F D_{\bar{m}}$, $p_{\bar{m}}(G)$ is continuous on its domain since composition of continuous partial functions, moreover:

$\left\langle f_{1}, \ldots, f_{n}\right\rangle \in \operatorname{dom}\left(p_{\bar{m}}(G)\right)$ iff

$\forall k \in \mathbb{N} . \exists[a, b] . b-a \leq 2^{-k} \wedge[a, b] \in G\left(e_{m_{1}}\left(f_{1}\right), \ldots e_{m_{n}}\left(f_{n}\right)\right) \quad$ iff

$\forall k \in \mathbb{N} . \exists[a, b] . b-a \leq 2^{-k} \wedge \exists d_{1} \ldots d_{n}$ finite.$f_{1} \in e_{m_{1}}^{-1}\left(\uparrow d_{1} \cap F D_{m_{1}}^{\dagger}\right) \wedge \ldots$

$\wedge f_{n} \in e_{m_{n}}^{-1}\left(\uparrow d_{n} \cap F D_{m_{n}}^{\dagger}\right) \wedge[a, b] \in G\left(d_{1} \ldots d_{n}\right) \quad$ iff

$\left\langle f_{1}, \ldots, f_{n}\right\rangle \in \bigcap_{k \in \mathbb{N}} \bigcup\left\{e_{m_{1}}^{-1}\left(\uparrow d_{1} \cap F D_{m_{1}}^{\dagger}\right) \times \ldots \times e_{m_{n}}^{-1}\left(\uparrow d_{n} \cap F D_{m_{n}}^{\dagger}\right) \mid\right.$

$d_{1} \ldots d_{n}$ finite $\left.\wedge \exists[a, b] . b-a \leq 2^{-k} \wedge[a, b] \in G\left(d_{1} \ldots d_{n}\right)\right\}$.

And obviously the set contained in the last expression is a countable intersection of open sets.

A similar and somewhat stronger result can be found in Weihrauch and Schreiber [WS81]. There, however, just first-order functions are considered and a different class of domains is used, namely, the $\omega$-algebraic cpo's with weight and distance.

It is now natural to extend the notion of computability to partial functions.

Definition 18 A partial function $f$ in $\mathbb{F}_{n}^{p}$ is computable if there is a computable element $g$ in $F D_{n}$ such that $f=p_{n}(g)$. A partial functional $F$ in $\mathbb{F}_{\bar{m}}^{p}$ is computable if there is a computable element $G$ in $F D_{\bar{m}}$ such that $F=p_{\bar{m}}(G)$.

So we have that every computable partial function (functional) is defined on a set which is the countable intersection of open sets and is continuous on its domain of definition. 


\section{Conclusions}

In this work we analysed the exact real number computations in functional programming languages. It is shown that domain theory can be usefully employed to carry on an analysis of computability on real numbers. A limitation of domain theory has been pointed out: using Scott-domain we cannot obtained a completely faithful representation of the real line. The main result presented in the article is a topological property of the computable functionals on real numbers: we show that every computable functional is continuous w.r.t. the compact-open topology on the functions space.

\section{Acknowledgements}

I thank Michael B. Smyth and Furio Honsell for many interesting and illuminating discussions. I would like to acknowledge also Thierry Coquand and Wilson de Olivera for their comments and the anonymous referees for their remarks.

\section{References}

[Abe80] O. Aberth. Computable analysis. MacGraw-Hill, New York, 1980.

[AJ94] S. Ambramsky and A. Jung. Domain theory. In S. Abramsky, D. M. Gabbay, and T. S. E. Maibaum, editors, Handbook of Logic in Computer Science, volume 3. Clarendom Press, 1994.

[Avi64] A. Avizienis. Binary-computable signed-digit arithmetic. In AFIPS Conference Proceedings 26,1, pages 663-672, 1964.

[BCRO86] H.-J. Boehm, R. Cartwright, M. Riggle, and M.J. O’Donell. Exact real arithmetic: a case study in higher order programming. In $A C M$ Symposium on lisp and functional programming, 1986.

[Bee85] M.J. Beeson. Foundation of constructive mathematics. SprigerVerlag, Berlin, 1985.

[Bis67] E. Bishop. Foundation of constructive analysis. McGraw-Hill, New York, 1967.

[Boe87] H.-J. Boehm. Constructive real interpretation of numerical programs. SIGPLAN Notice, 22, 7:214-221, July 87.

[Dug66] J. Dugundji. Topology. Allyn and Bacon, Boston, 1966.

[Gru88] K.E. Grue. Unrestricted lazy numerical algorithms. ., 1988.

[Grz57] A. Grzegorczyk. On the definition of computable real continuous functions. Fund. Math., 44:61-77, 1957. 
[Lac59] D. Lacombe. Quelques procédés de définitions en topologie recursif. In Constructivity in mathematics, pages 129-158. North-Holland, 1959.

[ML70] P. Martin-Löf. Note on Constructive Mathematics. Almqvist and Wiksell, Stockholm, 1970.

[Ric54] H.G. Rice. Recursive real numbers. In Proc. Amer. Math. Soc 5, pages 784-791, 1954.

[S9̈5] Ph. Sünderhauf. A faithful computational model of the real numbers. Theoret. Comput. Sci., 151(1):277-294, 1995.

[Sco70] Dana Scott. Outline of the mathematical theory of computation. In Proc. 4th Princeton Conference on Information Science, 1970.

[Smy76] Michael .B. Smyth. Effectively given domains. Theoret. Comp. Sci., $2: 257-274,1976$.

[Tur37] A.M. Turing. On computable numbers, with an application to the entscheidungs problem. In Proc. London Math. Soc. 42, pages 230$265,1937$.

[TvD88] A.S. Troelstra and D. van Dalen. Constructivism in Mathematics. North-Holland, Amsterdam, 1988.

[Vui88] J. Vuillemin. Exact real computer arithmetic with continued fraction. In Proc. A.C.M. conference on Lisp and functional Programming, pages 14-27, 1988.

[Wei95] K. Weihrauch. A simple introduction to computable analysis. Technical report, FernUniversität, Hagen, 1995.

[Wie80] E. Wiedmer. Computing with infinite objects. Theoret. Comp. Sci., 10:133-155, 1980.

[WS81] K. Weihrauch and U. Schreiber. Embeding metric spaces into cpo's. Theoret. Comp. Sci., 16:5-34, 1981. 